\title{
Estimation and Forecasting in Vector Autoregressive Moving Average Models for Rich Datasets
}

\author{
Gustavo Fruet Dias* \\ Department of Economics and Business Economics, Aarhus University \\ CREATES \\ George Kapetanios \\ King's College London
}

\begin{abstract}
We address the issue of modelling and forecasting macroeconomic variables using rich datasets by adopting the class of Vector Autoregressive Moving Average (VARMA) models. We overcome the estimation issue that arises with this class of models by implementing an iterative ordinary least squares (IOLS) estimator. We establish the consistency and asymptotic distribution of the estimator for weak and strong VARMA(p,q) models. Monte Carlo results show that IOLS is consistent and feasible for large systems, outperforming the MLE and other linear regression based efficient estimators under alternative scenarios. Our empirical application shows that VARMA models are feasible alternatives when forecasting with many predictors. We show that VARMA models outperform the $\operatorname{AR}(1), \operatorname{ARMA}(1,1)$, Bayesian VAR, and factor models, considering different model dimensions.
\end{abstract}

JEL classification numbers: C13, C32, C53, C63, E0

Keywords: VARMA, weak VARMA, Iterative ordinary least squares (IOLS) estimator, Asymptotic contraction mapping, Forecasting, Rich and Large datasets.

Acknowledgments: This paper previously circulated under the title "Forecasting Medium and Large Datasets with Vector Autoregressive Moving Average (VARMA) Models". We are indebted to Emmanuel Guerre, Cristina Scherrer and the seminar participants at Queen Mary (Econometrics Reading Group), the 14th Brazilian Time Series and Econometrics School (Gramado, Aug 2011), the Econometric Society Australasian Meeting (Adelaide, July 2011), the Royal Economic Society Annual Conference (Royal Holloway, University of London, April 2011) and the 4th International Conference on Computational and Financial Econometrics (London, Dec 2010).

Gustavo Fruet Dias acknowledges support from CREATES - Center for Research in Econometric Analysis of Time Series (DNRF78), funded by the Danish National Research Foundation. The usual disclaimer applies.

\footnotetext{
${ }^{*}$ Corresponding author at: Department of Economics and Business Economics, Aarhus University, Fuglesangs Allé 4, 8210 Aarhus V, Denmark. E-mail: gdias@econ.au.dk.
} 


\section{Introduction}

The use of large arrays of economic indicators to forecast key macroeconomic variables has become very popular recently. Economic agents consider a wide range of information when they construct their expectations about the behaviour of macroeconomic variables such as interest rates, industrial production, and inflation. In the past several years, this information has become more widely available through a large number of indicators that aim to describe different sectors and fundamentals from the whole economy. To improve forecast accuracy, large sized datasets that attempt to replicate the set of information used by agents to make their decisions are incorporated into econometric models.

For the past twenty years, macroeconomic variables have been forecasted using vector autoregression (VAR) models. This type of model performs well when the number of variables in the system is relatively small. When the number of variables increases, however, the performance of VAR forecasts deteriorates very fast, generating the so-called "curse of dimensionality". In this paper, we propose the use of vector autoregressive moving average (VARMA) models, estimated with the iterative ordinary least squares (IOLS) estimator, as a feasible method to address the "curse of dimensionality" on medium and large sized datasets and improve forecast accuracy of macroeconomic variables. To the best of our knowledge, the VARMA methodology has never been applied to medium and large sized datasets, as we do in this paper.

VARMA models have been studied for the past thirty years, but they have never been as popular as VAR models because of estimation and specification issues. Despite having attractive theoretical properties, estimation of VARMA models remains a challenge. Linear estimators (Hannan and Rissanen (1982), Hannan and Kavalieris (1984), Dufour and Jouini (2014), among others) and Bayesian methods (Chan, Eisenstat, and Koop (2016)) have been proposed in the literature as a way to overcome the numerical difficulties posed by the efficient maximum-likelihood estimator. We address the estimation issue and hence contribute to this literature by proposing the use of the IOLS estimator that is feasible for high-dimensional VARMA models.

Other methodologies have been proposed in the literature to deal with the "curse of dimensionality". We can divide them mainly in two groups of models. The first aims to overcome the dimensionality issue by imposing restrictions on the parameter matrices of a standard VAR model. Among the many important contributions from this field, we 
point out the following classes of models: Bayesian VAR (BVAR) (De Mol, Giannone, and Reichlin (2008) and Bańbura, Giannone, and Reichlin (2010)); Ridge (De Mol, Giannone, and Reichlin (2008)); reduced rank VAR (Carriero, Kapetanios, and Marcellino (2011)); and Lasso (De Mol, Giannone, and Reichlin (2008) and Tibshirani (1996)). The second group of models dealing with the "curse of dimensionality" reduces the dimension of the dataset by constructing summary proxies from the large dataset. Chief among these models is the class of factor models. The seminal works in this area are Forni, Hallin, Lippi, and Reichlin (2000) and Stock and Watson (2002a,b). Common factor models improve forecast accuracy and produce theoretically well-behaved impulse response functions, as reported by De Mol, Giannone, and Reichlin (2008) and Bernanke, Boivin, and Eliasz (2005).

VARMA models are able to capture two important features from these two groups of models. The first is the reduction of the model dimensionality, achieved by setting some elements of the parameter matrices to zero following uniqueness requirements. This produces parameter matrices which are not full rank, resembling the reduced rank VAR model of Carriero, Kapetanios, and Marcellino (2011). Furthermore, VARMA models parsimoniously account for sample correlation profiles of different shapes than the geometrically declining sinusoids associated with the VAR models. The second is the relationship between VARMA models and factor representations of a vector stochastic process. If the latent common factors follow either a finite VAR or VARMA processes, then the observed series will have a VARMA representation (Dufour and Stevanović (2013)). This fact reinforces the use of VARMA models as a suitable framework to forecast key macroeconomic variables using potentially many predictors. Additionally, VARMA models are closed under linear transformation and marginalization (see Lütkepohl (2007, Section 11.6.1)), providing additional flexibility and potentially better forecast performance.

With regard to the theory, we establish the consistency and asymptotic distribution of the IOLS estimator for the weak and strong VARMA $(\mathrm{p}, \mathrm{q})$ models. Strong VARMA models have innovations that are independently identically distributed (i.i.d.), while the disturbances of the weak VARMA models are only uncorrelated. Our asymptotic results are obtained under mild assumptions using the asymptotic contraction mapping framework of Dominitz and Sherman (2005). We conduct an extensive Monte Carlo study, considering different system dimensions, sample sizes, weak and strong innovations, eigenvalues associated with the parameter matrices, and Kronecker indices specifications. We show that the 
IOLS estimator has a good finite sample performance when compared to alternative estimators, such as the efficient maximum-likelihood (MLE) estimator, and important linear competitors. Specifically, the IOLS estimator performs well in a variety of scenarios such as small sample size; the eigenvalues associated with the parameter matrices are near-to-zero; and high-dimensional systems.

In the empirical part of the paper, we focus on forecasting three key macroeconomic variables: industrial production, interest rate, and CPI inflation using potentially large sized datasets. As in Carriero, Kapetanios, and Marcellino (2011), we use the 52 US macroeconomic variables taken from the dataset provided by Stock and Watson (2006) to construct systems with five different dimensions: 3, 10, 20, 40, and 52 variables. By doing that, we are able to evaluate the tradeoff between forecast gains by incorporating more information (large sized datasets) and the estimation cost associated with it. Additionally, the different system dimensions play the role of robustness check. We show that VARMA models are strong competitors and produce more accurate forecasts than the benchmark models (AR(1), ARMA(1,1), BVAR, and factor models) in different occasions. This conclusion holds for different system sizes and horizons.

The paper is structured as follows. In Section 2, we discuss the properties and identification of VARMA models and derive the IOLS estimator. In Section 3, we establish the consistency and asymptotic distribution of the IOLS estimator considering the general weak and strong VARMA(p,q) models. In Section 4, we address the consistency and efficiency of VARMA models estimated with the IOLS procedure through a Monte Carlo study. In Section 5, we display the results of our empirical application. The proofs and tables are relegated to an Appendix. An online Supplement presents proofs for the auxiliary Lemmas, the entire set of tables with results for the Monte Carlo and the empirical studies, and further discussion on selected topics.

\section{VARMA Models, Identification, and Estimation Proce- dures}

Our interest lies in modelling and forecasting key elements of the $K$-dimensional vector process $Y_{t}=\left(y_{1, t}, y_{2, t}, \ldots, y_{K, t}\right)^{\prime}$, where $K$ is allowed to be large. We assume, as a baseline 
model, a general nonstandard $\operatorname{VARMA}(\mathrm{p}, \mathrm{q})$ model where the means have been removed, ${ }^{1}$

$A_{0} Y_{t}=A_{1} Y_{t-1}+A_{2} Y_{t-2}+\ldots+A_{p} Y_{t-p}+M_{0} U_{t}+M_{1} U_{t-1}+\ldots+M_{q} U_{t-q}$,

where the disturbances $U_{t}=\left(u_{1, t}, u_{2, t}, \ldots, u_{K, t}\right)^{\prime}$ are assumed to be a zero-mean sequence of uncorrelated random variables (weak white noise process) with a nonsingular $(K \times K)$ covariance matrix $\Sigma_{u}$, and $Y$ has dimension $(K \times 1)$. Our baseline model is assumed to be stable and invertible. ${ }^{2}$

\subsection{Identification and Uniqueness}

The issue of identifying unique parameterizations of VARMA models has been an important topic of study in econometrics and statistics (Hannan (1969, 1976), Akaike (1974, 1976), Hannan and Kavalieris (1984), Hannan and Deistler (1988), among others). This follows because nonstandard VARMA models require restrictions on the parameter matrices to ensure that the model is uniquely identified. To formally define uniqueness of a VARMA representation, define the lag polynomials $A(L)=A_{0}-A_{1} L-A_{2} L^{2}-\ldots-A_{p} L^{p}$ and $M(L)=M_{0}+M_{1} L+M_{2} L^{2}+\ldots+M_{q} L^{q}$, where $L$ is the usual lag operator. More specifically, we say that a model is unique if there is only one pair of stable and invertible polynomials $A(L)$ and $M(L)$, respectively, which satisfies the canonical MA representation

$Y_{t}=A(L)^{-1} M(L)=\Theta(L) U_{t}=\sum_{i=0}^{\infty} \Theta_{i} U_{t-i}$

for a given $\Theta(L)$ operator. In contrast to the reduced form VAR models, setting $A_{0}=$ $M_{0}=I_{K}$ is not sufficient to ensure a unique VARMA representation. Uniqueness of (1) is guaranteed by imposing restrictions on the $A(L)$ and $M(L)$ operators in that these operators are unique left-coprime, i.e. the only feasible left divisor of $[A(L): M(L)]$ is the unimodular operator given by the identity matrix. ${ }^{3}$

A number of different strategies can be implemented to obtain unique VARMA representations, such as the extended scalar component approach of Athanasopoulos and Vahid

\footnotetext{
${ }^{1}$ We adopt the terminology in Lütkepohl, 2007, p. 448 and call a VARMA representation nonstandard when $A_{0}$ and $M_{0}$ are allowed to be nonidentity invertible matrices. If $A_{0}=M_{0}=I_{K}$, we call it a standard VARMA model.

${ }^{2} \mathrm{~A}$ general $\operatorname{VARMA}(\mathrm{p}, \mathrm{q})$ is considered stable and invertible if $\operatorname{det}\left(A_{0}-A_{1} z-A_{2} z^{2}-\ldots-A_{p} z^{p}\right) \neq 0$ for $|z| \leq 1$ and $\operatorname{det}\left(M_{0}+M_{1} z+M_{2} z^{2}+\ldots+M_{q} z^{q}\right) \neq 0$ for $|z| \leq 1$ hold, respectively.

${ }^{3} C(\bar{L})$ is an unimodular operator if $\operatorname{det}(C(L))$ is a nonzero constant that does not depend on $L$.
} 
(2008), the final equations form (see Lütkepohl, 2007, pg.362) and the Echelon form transformation (Hannan and Kavalieris (1984), Poskitt (1992), Lütkepohl and Poskitt (1996), among others). Although Athanasopoulos, Poskitt, and Vahid (2012) show that scalar components perform slightly better than the Echelon form methodology in empirical exercises, the authors argue that the latter has the advantage of having a simpler identification procedure. More specifically, the Echelon form identification strategy can be fully automated and provides a more parsimonious parametrization, when compared to final equations. These are highly desired features when modelling medium and large systems. In this paper, we implement the Echelon form transformation as a way to impose uniqueness in both Monte Carlo and empirical applications. Nevertheless, because the three identification strategies (scalar components, final equations form, and Echelon form) impose uniqueness through a set of linear restrictions on the $A(L)$ and $M(L)$ operators, the IOLS estimator can be directly implemented no matter which identification strategy the researcher chooses.

A general VARMA model such as the one stated in (1) is considered to be in its Echelon form if the conditions stated in equations (3), (4), (5), and (6) are satisfied (see Lütkepohl, 2007, p. 452 and Lütkepohl and Poskitt (1996) for more details):

$p_{k i}:=\left\{\begin{array}{c}\min \left(p_{k}+1, p_{i}\right) \text { for } k \geq i \\ \min \left(p_{k}, p_{i}\right) \text { for } k<i,\end{array} \quad\right.$ for $k, i=1, \ldots, K$,

$\alpha_{k k}(L)=1-\sum_{j=1}^{p_{k}} \alpha_{k k, j} L^{j} \quad$ for $k=1, \ldots, K$,

$\alpha_{k i}(L)=-\sum_{j=p_{k}-p_{k i}+1}^{p_{k}} \alpha_{k i, j} L^{j} \quad$ for $k \neq i$

$m_{k i}(L)=\sum_{j=0}^{p_{k}} m_{k i, j} L^{j}, \quad$ for $k, i=1, \ldots, K \quad$ with $\quad M_{0}=A_{0}$,

where $A(L)=\left[\alpha_{k i}\right]_{k, i=1, \ldots, K}$ and $M(L)=\left[m_{k i}\right]_{k, i=1, \ldots, K}$ are, respectively, the operators from the autoregressive and moving average components of the VARMA process; the $p_{k i}$ numbers specify the free coefficients in the operator $\alpha_{k i}(L)$ for $i \neq k$ from the $A(L)$ polynomial; and the arguments $p_{k}$ with $k=1, \ldots, K$ are Kronecker indices which specify the maximum degrees in each row of both $A(L)$ and $M(L)$ polynomials. We define $\mathbf{p}=\left(p_{1}, p_{2}, \ldots, p_{K}\right)^{\prime}$ as the vector collecting the Kronecker indices (Section 5.1 discusses alternative procedures to choose the Kronecker indices). 
The Echelon form formulae in (3) to (6) deliver the necessary and sufficient restrictions for the unique identification of the VARMA models (Hannan and Deistler, 1988, Chapter 2). Notably, the Echelon representation allows the baseline model in (1) to be rewritten as

$Y_{t}=\left(I_{K}-A_{0}\right)\left(Y_{t}-U_{t}\right)+A_{1} Y_{t-1}+A_{2} Y_{t-2}+\ldots+A_{p} Y_{t-p}+U_{t}+M_{1} U_{t-1}+\ldots+M_{q} U_{t-q}$,

where $\left(Y_{t}-U_{t}\right)=A_{0}^{-1}\left\{\sum_{i=1}^{p} A_{i} Y_{t-i}+\sum_{i=1}^{q} M_{i} U_{t-i}\right\}$ is uncorrelated with $U_{t}$. A compact notation of (7) reads

$\operatorname{vec}(Y)=\left(X^{\prime} \otimes I_{K}\right) \operatorname{vec}(B)+\operatorname{vec}(U)$,

where $B=\left[\left(I_{K}-A_{0}\right), A_{1}, \ldots, A_{p}, M_{1}, \ldots, M_{q}\right]$ with dimension $(K \times K(p+q+1)) ; X=$ $\left(X_{\bar{q}+1}, \ldots, X_{T}\right)$ is the matrix of regressors with dimension $(K(p+q+1) \times T-\bar{q})$, where $\bar{q}=\max \{p, q\}$ and $X_{t}=\operatorname{vec}\left(Y_{t}-U_{t}, Y_{t-1}, \ldots, Y_{t-p}, U_{t-1}, \ldots, U_{t-q}\right) ; Y=\left(Y_{\bar{q}+1}, \ldots, Y_{T}\right)$ has dimension $(K \times T-\bar{q})$; and $U=\left(U_{\bar{q}+1}, \ldots, U_{T}\right)$ is a $(K \times T-\bar{q})$ matrix of disturbances. Note that by setting the dimensions of the $X$ and $Y$ matrices as $(K(p+q+1) \times T-\bar{q})$ and $(K \times T-\bar{q})$, respectively, we explicitly highlight the fact that we lose $\bar{q}$ observations in finite sample. We obtain the free parameters in the model (excluding the distinct covariance parameters) by rewriting vec $(B)$ into the product of a $\left(K^{2}(p+q+1) \times n\right)$ deterministic matrix $R$ and a $(n \times 1)$ vector $\beta$,

$\operatorname{vec}(Y)=\left(X^{\prime} \otimes I_{K}\right) R \beta+\operatorname{vec}(U)$,

where $n$ denotes the number of free parameters in $B$, and the $(n \times 1)$ vector $\beta$ concatenates these parameters. The Echelon restrictions imply a unique full-rank column matrix $R$ and, provided that $\Sigma_{u}$ is nonsingular, ensure that $\operatorname{rank}\left\{R^{\prime}\left[\mathbb{E}\left(X_{t} X_{t}^{\prime}\right) \otimes I_{K}\right] R\right\}=n(\mathrm{Du}-$ four and Jouini $(2005,2014)$ ). It is still possible to impose additional zero restrictions (data dependent) while keeping the model uniquely identified and hence obtain a more parsimonious model (see Lütkepohl and Poskitt, 1996, p. 73). This follows because a Kronecker index only gives the maximum row degree, and hence not all operators in the $k^{\text {th }}$ row of $[A(L): M(L)]$ need to have the same degree as $p_{k}$. As an example, consider a $\operatorname{VARMA}(1,1)$ model with $K=3$ and $\mathbf{p}=(1,0,0)^{\prime}$. The resulting VARMA representation expressed in 
Echelon form reads

$$
\left(\begin{array}{ccc}
1 & 0 & 0 \\
a_{21,0} & 1 & 0 \\
a_{31,0} & 0 & 1
\end{array}\right) Y_{t}=\left(\begin{array}{ccc}
a_{11,1} & 0 & 0 \\
0 & 0 & 0 \\
0 & 0 & 0
\end{array}\right) Y_{t-1}+\left(\begin{array}{ccc}
1 & 0 & 0 \\
a_{21,0} & 1 & 0 \\
a_{31,0} & 0 & 1
\end{array}\right) U_{t}+\left(\begin{array}{ccc}
m_{11,1} & m_{12,1} & m_{13,1} \\
0 & 0 & 0 \\
0 & 0 & 0
\end{array}\right) U_{t-1}
$$

While the zero restrictions imposed in (10) are those required to meet the restriction imposed by the canonical structure, further restrictions that simplify the model could be added (e.g. $a_{21,0}=a_{31,0}=m_{12,1}=m_{13,1}=0$ ) without affecting the uniqueness of the VARMA representation. Throughout this entire study, we refrain from adding these additional restrictions and restrict ourselves to the ones implied from (3) to (6). Furthermore, we always consider the Echelon forms that yield VARMA models that cannot be partitioned into smaller independent systems.

Finally, it is important to note that the VARMA representation in (1) is not a structural VARMA (SVARMA) model in its classical definition (see Gourieroux and Monfort (2015)), because (1) is not necessarily driven by independent (or uncorrelated) shocks. To construct impulse response functions, which depend on the structural shocks, additional identification restrictions to the ones required for uniqueness are necessary. In particular, as noted by Gourieroux and Monfort (2015), the structural shocks can usually be derived by imposing restrictions either on the contemporaneous correlation among the innovations in (1) (see the so-called B-model in Lütkepohl, 2007, p. 362 and the general identification theory in Rubio-Ramírez, Waggoner, and Zha (2010)), or on the long-run impact matrix of the shocks (Blanchard and Quah (1989)), or by imposing sign restrictions on some impulse response functions (Uhlig (2005)). This identification issue is common to both VARMA and VAR models and has been primarily explored in the context of VAR models.

\subsection{Estimation}

VARMA models, similar to their univariate (ARMA model) counterparts, are usually estimated using MLE procedures. Provided that the model in (1) is uniquely identified and disturbances $U_{t}$ are normally distributed, MLE delivers consistent and efficient estimators. Although MLE seems to be very powerful at first glance, it presents serious problems when dealing with medium and large sized datasets. We overcome this issue by implementing an 
IOLS procedure in the spirit of Spliid (1983), Hannan and McDougall (1988) and Kapetan$\operatorname{ios}(2003)$.

The IOLS framework consists of computing ordinary least squares (OLS) estimates of the parameters using estimates of the latent regressors. These regressors are computed recursively at each iteration using the OLS estimates. Using the invertibility condition, we can express a finite VARMA model as an infinite VAR, $Y_{t}=\sum_{i=1}^{\infty} \Pi_{i} Y_{t-i}+U_{t}$. We compute consistent estimates of $U_{t}$, denoted as $\widehat{U}_{t}^{0}$, by truncating the infinite VAR representation using some lag order, $\tilde{p}$, that minimizes some criterion. Following the results in $\mathrm{Ng}$ and Perron (1995) and Dufour and Jouini (2005), choosing $\tilde{p}$ proportional to $\ln (T)$ delivers consistent estimates of $U_{t}$, so that $\widehat{U}_{t}^{0}=Y_{t}-\sum_{i=1}^{\tilde{p}} \widehat{\Pi}_{i} Y_{t-i} \cdot{ }^{4}$ Substitute $\widehat{U}_{t}^{0}$ into the matrix $X$ in $(9)$ and denote it $\widehat{X}^{0}$. Note that both $\widehat{X}^{0}$ and vec $(Y)$ change their dimensions to $(K(p+q+1) \times T-\bar{q}-\tilde{p})$ and $(K(T-\bar{q}-\tilde{p}) \times 1)$, respectively. This happens exclusively on this first iteration, because $\tilde{p}$ observations are lost on the $\operatorname{VAR}(\tilde{p})$ approximation of $U_{t}$. The first iteration of the IOLS estimator is obtained by computing the OLS estimator from the modified version of (9),

$\widehat{\beta}^{1}=\left[R^{\prime}\left(\widehat{X}^{0} \widehat{X}^{0 \prime} \otimes I_{K}\right) R\right]^{-1} R^{\prime}\left(\widehat{X}^{0} \otimes I_{K}\right) \operatorname{vec}(Y)$.

It is relevant to highlight that the first step of the IOLS estimator is the two-stage HannanRissanen (HR) algorithm formulated in Hannan and Rissanen (1982), and for which Dufour and Jouini (2005) show the consistency and asymptotic distribution.

We are now in a position to use $\widehat{\beta}^{1}$ to recover the parameter matrices $\widehat{A}_{0}^{1}, \ldots, \widehat{A}_{p}^{1}$, $\widehat{M}_{1}^{1}, \ldots, \widehat{M}_{q}^{1}$ and a new set of residuals $\widehat{U}^{1}=\left(\widehat{U}_{1}^{1}, \widehat{U}_{2}^{1}, \ldots, \widehat{U}_{T}^{1}\right)$ by recursively applying

$\widehat{U}_{t}^{1}=Y_{t}-\left[\widehat{A}_{0}^{1}\right]^{-1}\left[\widehat{A}_{1}^{1} Y_{t-1}-\ldots-\widehat{A}_{p}^{1} Y_{t-p}-\widehat{M}_{1}^{1} \widehat{U}_{t-1}^{1}-\ldots-\widehat{M}_{q}^{1} \widehat{U}_{t-q}^{1}\right]$, for $t=1, \ldots, T$,

where $Y_{t-\ell}=\widehat{U}_{t-\ell}^{1}=0$ for all $\ell \geq t$. Setting the initial values to zero when computing the residuals recursively on any iteration is asymptotically negligible (see Lemma 2). Note that the superscript on the parameter matrices refers to the iteration in which those parameters are computed, and the subscript is the usual lag order. We compute the second iteration of the IOLS procedure by plugging $\widehat{U}_{t}^{1}$ into $(9)$ yielding $\widehat{X}^{1}$. Note that $\widehat{X}^{1}=\left(\widehat{X}_{\bar{q}+1}^{1}, \ldots, \widehat{X}_{T}^{1}\right)$,

\footnotetext{
${ }^{4}$ Lemmas 4.1 and 4.2 in $\mathrm{Ng}$ and Perron (1995) show that determining the truncation lag proportional to $\ln (T)$ guarantees that the difference between the residuals from the truncated VAR model and the ones obtained from the infinite VAR process is $o_{p}\left(T^{-1 / 2}\right)$ uniformly in $\tilde{p}$.
} 
where $\widehat{X}_{t}^{1}=\left[Y_{t}-\widehat{U}_{t}^{1}, Y_{t-1}, \ldots, Y_{t-p}, \widehat{U}_{t-1}^{1}, \ldots, \widehat{U}_{t-q}^{1}\right]^{\prime}$, is a function of the estimates obtained

in the first iteration: $\widehat{\beta}^{1}$. Similarly as in (11), we obtain $\widehat{\beta}^{2}$ and its correspondent set of residuals recursively as in (12). The $j^{\text {th }}$ iteration of the IOLS estimator is thus given by

$\widehat{\beta}^{j}=\left[R^{\prime}\left(\widehat{X}^{j-1} \widehat{X}^{j-1 \prime} \otimes I_{K}\right) R\right]^{-1} R^{\prime}\left(\widehat{X}^{j-1} \otimes I_{K}\right) \operatorname{vec}(Y)$.

We stop the IOLS algorithm when estimates of $\beta$ converge. We assume that $\widehat{\beta}^{j}$ converges if $\left\|\widehat{U}^{j}-\widehat{U}^{j-1}\right\| \leq \epsilon$ holds from some exogenously defined criterion $\epsilon$, where $\|\cdot\|$ accounts for the Frobenius norm. If the IOLS fails to converge, we adopt the consistent HR estimator, $\widehat{\beta}^{1}$, given in (11). The maximum number of iterations is set to 1,000 and $\epsilon=10^{-5}$. We also notice from our simulations that sample size, number of free parameters, system dimension, and the values of $\beta$ play an important role on the convergence rates of the IOLS estimator. In general, we find that convergence rates increase monotonically with $T$ (see discussion in Sections 3 and 4$)$.

\section{Theoretical Properties}

This section provides theoretical results regarding the consistency and the asymptotic distribution of the IOLS estimator. A previous attempt to establish these results have been made by Hannan and McDougall (1988). They prove the consistency of the IOLS estimator considering the univariate $\operatorname{ARMA}(1,1)$ specification, but no formal result is provided for the asymptotic normality. Overall, this section differs from the work of Hannan and McDougall (1988) in important ways. First, we derive the consistency and the asymptotic normality for the general weak and strong $\operatorname{VARMA}(\mathrm{p}, \mathrm{q})$ models; and second, our theory explicitly accounts for the effects of setting initial values equal to zero when updating the residuals on each iteration.

Similarly as in Section 2, we define our baseline weak VARMA(p,q) model expressed in its Echelon form as in (14) and its more compact notation in (15):

$A_{0} Y_{t}=A_{1} Y_{t-1}+A_{2} Y_{t-2}+\ldots+A_{p} Y_{t-p}+A_{0} U_{t}+M_{1} U_{t-1}+\ldots+M_{q} U_{t-q}$,

$Y_{t}=\left(X_{t}^{\prime} \otimes I_{K}\right) R \beta+U_{t}$,

where $U_{t}$ is a sequence of uncorrelated random variables. 
We base our asymptotic results on the general theory for iterative estimators developed by Dominitz and Sherman (2005). ${ }^{5}$ Their approach relies on the concept of the Asymptotic Contraction Mapping $(\mathrm{ACM})$. Denote $(\mathbb{B}, d)$ as a metric space where $\mathbb{B}$ is the closed ball centered in $\beta$ and $d$ is a distance function; $(\Omega, \mathcal{A}, \mathbb{P})$ is a probability space, where $\Omega$ is a sample space, $\mathcal{A}$ is a $\sigma$-field of subsets of $\Omega$ and $\mathbb{P}$ is the probability measure on $\mathcal{A}$; and $K_{T}^{\omega}($.$) is a function defined on \mathbb{B}$, with $\omega \in \Omega$. From the definition in Dominitz and Sherman, 2005, p. 841, "The collection $\left\{K_{T}^{\omega}():. T \geq 1, \omega \in \Omega\right\}$ is an $A C M$ on $(\mathbb{B}, d)$ if there exist a constant $c \in[0,1)$ that does not depend on $T$ or $\omega$, and sets $\left\{\mathcal{A}_{T}\right\}$ with each $\mathcal{A}_{T} \subseteq \Omega$ and $\mathbb{P} \mathcal{A}_{T} \rightarrow 1$ as $T \rightarrow \infty$, such that for each $\omega \in \mathcal{A}_{T}, K_{T}^{\omega}($.$) maps \mathbb{B}$ to itself and for all $x, y \in \mathbb{B}, d\left(K_{T}^{\omega}(x), K_{T}^{\omega}(y)\right) \leq c d(x, y)$ ". As pointed out by Dominitz and Sherman (2005), if a collection is an $\mathrm{ACM}$, then it will have a unique fixed point in $(\mathbb{B}, d)$, where the fixed point now depends on the sample characteristics, i.e. $T$ and $\omega$. Additionally, their ACM definition nests the case where the population mapping is a fixed deterministic function (Dominitz and Sherman, 2005, p. 840).

Definition 1 (General Mapping) We define the sample mapping $\widehat{N}_{T}\left(\widehat{\beta}^{j}\right)$ and its population counterpart $N\left(\beta^{j}\right)$ as follows:

$$
\begin{aligned}
\text { i. } \widehat{\beta}^{j+1} & =\widehat{N}_{T}\left(\widehat{\beta}^{j}\right)=\left[\frac{1}{T-\bar{q}} \sum_{t=\bar{q}+1}^{T} \widetilde{X}_{t}^{j \prime} \widetilde{X}_{t}^{j}\right]^{-1}\left[\frac{1}{T-\bar{q}} \sum_{t=\bar{q}+1}^{T} \widetilde{X}_{t}^{j \prime} Y_{t}\right], \\
\text { ii. } \beta^{j+1} & =N\left(\beta^{j}\right)=\mathbb{E}\left[\widetilde{X}_{\infty, t}^{j \prime} \widetilde{X}_{\infty, t}^{j}\right]^{-1} \mathbb{E}\left[\widetilde{X}_{\infty, t}^{j \prime} Y_{t}\right],
\end{aligned}
$$

where $\tilde{X}_{t}^{j}=\left[\left(\widehat{X}_{t}^{j \prime} \otimes I_{K}\right) R\right]$ and $\widetilde{X}_{\infty, t}^{j}=\left[\left(X_{\infty, t}^{j \prime} \otimes I_{K}\right) R\right]$ have dimensions $(K \times n)$ and denote the regressors computed on the $j^{\text {th }}$ iteration; $n$ is the number of free parameters (excluding the distinct covariance parameters) in the model; $\widehat{X}_{t}^{j}=\operatorname{vec}\left(Y_{t}-\right.$ $\left.\widehat{U}_{t}^{j}, Y_{t-1}, \ldots, Y_{t-p}, \widehat{U}_{t-1}^{j}, \ldots, \widehat{U}_{t-q}^{j}\right), \quad X_{\infty, t}^{j}=\operatorname{vec}\left(Y_{t}-U_{t}^{j}, Y_{t-1}, \ldots, Y_{t-p}, U_{t-1}^{j}, \ldots, U_{t-q}^{j}\right) ;$ $\bar{q}=\max \{p, q\}$; and the $(n \times 1)$ vectors $\widehat{\beta}^{j}$ and $\beta^{j}$ stack the estimates obtained from the sample and population mappings, respectively. Notably, $\widehat{N}_{T}\left(\widehat{\beta}^{j}\right)$ and its population counterpart map from $\mathbb{R}^{n}$ to $\mathbb{R}^{n}$. The sample and population mappings differ in two important ways. First, the population mapping is a deterministic function, whereas its sample counterpart is stochastic. Second, $\widehat{U}_{t}^{j}$ is obtained recursively by

$$
\widehat{U}_{t}^{j}=Y_{t}-\left[\widehat{A}_{0}^{j}\right]^{-1}\left[\widehat{A}_{1}^{j} Y_{t-1}-\ldots-\widehat{A}_{p}^{j} Y_{t-p}-\widehat{M}_{1}^{j} \widehat{U}_{t-1}^{j}-\ldots-\widehat{M}_{q}^{j} \widehat{U}_{t-q}^{j}\right], \text { for } t=1, \ldots, T,
$$

\footnotetext{
${ }^{5}$ Pastorello, Patilea, and Renault (2003) develop a similar general asymptotic theory for iterative estimators that also rests on the contracting property and hence on the unique fixed point condition.
} 
where $Y_{t-\ell}=\widehat{U}_{t-\ell}^{j}=0$ for all $\ell \geq t$, while $U_{t}^{j}$ is also computed recursively in the same fashion as (16), but assumes the pre-sample values are known, i.e. $Y_{t-\ell}$ and $U_{t-\ell}^{j}$ are known for all $\ell \geq t$. Note that $N(\beta)=\beta$, which implies that when evaluated on the true vector of parameters, the population mapping maps the vector $\beta$ to itself. This implies that if the population mapping is an ACM then $\beta$ is a unique fixed point of $N(\beta)$ (see Dominitz and Sherman, 2005, p. 841).

For theoretical reasons, such that we can formally handle the effect of initial values when computing the residuals recursively, define the infeasible sample mapping as

$\breve{\beta}^{j+1}=\breve{N}_{T}\left(\breve{\beta}^{j}\right)=\left[\frac{1}{T-\bar{q}} \sum_{t=\bar{q}+1}^{T} \widetilde{X}_{\infty, t}^{j \prime} \widetilde{X}_{\infty, t}^{j}\right]^{-1}\left[\frac{1}{T-\bar{q}} \sum_{t=\bar{q}+1}^{T} \widetilde{X}_{\infty, t}^{j \prime} Y_{t}\right]$.

The infeasible sample mapping in (17) differs from the sample mapping because it is a function of $U_{t}^{j}$ rather than $\widehat{U}_{t}^{j}$. This is a stochastic version of the population mapping and it is extensively used when deriving the consistency and the asymptotic normality of the IOLS estimator. Lemma 2 shows that, when evaluated at the same vector of estimates, $\widehat{N}_{T}\left(\widehat{\beta}^{j}\right)$ converges uniformly to its infeasible counterpart as $T \longrightarrow \infty$, which implies that setting the starting values to zero in (16) does not matter asymptotically.

To formally derive the consistency and asymptotic normality of the IOLS estimator, we impose the following assumptions:

B.1 (Stability, Invertibility, and Uniqueness) Let $Y_{t}$ be a stable and invertible $K$ dimensional $\operatorname{VARMA}(\mathrm{p}, \mathrm{q})$ process. Moreover, assume $Y_{t}$ is uniquely identified and expressed in Echelon form as in (14) with known Kronecker indices.

B.2 (Disturbances - strong mixing) Let $U_{t}$ be a $(K \times 1)$ vector of innovations with $K \geq 1$. The disturbances $U_{t}$ are strictly stationary with $\mathbb{E}\left(U_{t}\right)=0, \operatorname{Var}\left(U_{t}\right)=\Sigma_{u}$, $\operatorname{Cov}\left(U_{t-i}, U_{t-j}\right)=0$ for all $i \neq j$ and satisfy the following two conditions:

i. $\mathbb{E}\left|U_{t}\right|^{4+2 \nu}<\infty$,

ii. $\sum_{\kappa=0}^{\infty}\left\{\alpha_{u}(\kappa)\right\}^{\nu /(2+\nu)}<\infty$, for some $\nu>0$,

where $\alpha_{u}(l)=\sup _{\substack{\mathcal{D} \in \sigma\left(U_{i}, i \geq t+l\right) \\ \mathcal{C} \in \sigma\left(U_{i}, i \leq t\right)}}|\operatorname{Pr}(\mathcal{C} \cap \mathcal{D})-\operatorname{Pr}(\mathcal{C}) \operatorname{Pr}(\mathcal{D})|$ are strong mixing coefficients of order $l \geq 1$, with $\sigma\left(U_{i}, i \leq t\right)$ and $\sigma\left(U_{i}, i \geq t+l\right)$ being the $\sigma$-fields generated by $\left\{U_{i}: i \leq t\right\}$ and $\left\{U_{i}: i \geq t+l\right\}$, respectively. 
B.3 (Contraction and Stochastic Equicontinuity) Define the $(n \times n)$ infeasible sample gradient as $\breve{V}_{T}\left(\breve{\beta}^{j}\right)=\frac{\partial \breve{N}_{T}\left(\breve{\beta}^{j}\right)}{\partial \breve{\beta}^{j^{\prime}}}$ and its population counterpart as $V\left(\beta^{j}\right)=\frac{\partial N\left(\beta^{j}\right)}{\partial \beta^{j^{\prime}}}$; and $\mathbb{B}$ as the closed ball centered at $\beta$ satisfying invertibility and stability conditions in Assumption B.1. Assume that the following hold:

i. The maximum eigenvalue associated with $V(\beta)=\left.\frac{\partial N\left(\beta^{j}\right)}{\partial \beta^{j}}\right|_{\beta}$ is smaller than one in absolute value.

ii. $\sup _{\phi \in \mathbb{B}}\left\|\breve{V}_{T}(\phi)\right\|=O_{p}(1)$, with $\phi \in \mathbb{B}$.

Assumption B.1 provides the general regularity conditions governing the $\operatorname{VARMA}(\mathrm{p}, \mathrm{q})$ model. Assumption B.2 establishes the mixing conditions which satisfy the weak VARMA definition. Notably, these mixing conditions are valid for a wide range of nonlinear models that allow weak VARMA representations (Francq and Zakoian (1998), Francq, Roy, and Zakoian (2005) and Francq and Zakoian (2005)). Item i. in Assumption B.3 suffices to guarantee that the IOLS mapping is an $\mathrm{ACM}$ on $\left(\mathbb{B}, E_{n}\right)$. Lemma 1 provides the sample counterpart of this result, making it possible to verify on every iteration whether the sample mapping is an ACM. Albeit the result in Lemma 1 is computationally easy to obtain, we could not pin down the eigenvalues of the population counterpart of Lemma 1 solely as function of the parameters matrices eigenvalues. Numerical simulation indicates that the maximum eigenvalue of $V(\beta)$ depends on the elements of the parameters matrices in (14) rather than on their eigenvalues. Furthermore, we note that the population mapping is not an ACM if some of the eigenvalues of the AR and MA components have opposite signs and are close to one in absolute value.

Define $Z=\left[R^{\prime}\left(H \otimes I_{K}\right) R\right]^{-1}, H=\operatorname{plim}\left[\frac{1}{T} \sum_{t=\bar{q}+1}^{T} X_{t} X_{t}^{\prime}\right], J=\left[I_{n}-V(\beta)\right]^{-1}$, and $\mathcal{I}=\sum_{\ell=-\infty}^{\infty} \mathbb{E}\left\{\left[R^{\prime}\left(X_{t} \otimes I_{K}\right) U_{t}\right]\left[R^{\prime}\left(X_{t-\ell} \otimes I_{K}\right) U_{t-\ell}\right]^{\prime}\right\}$. Theorem 1 gives the consistency and asymptotic distribution of the IOLS estimator for the general weak VARMA(p,q) model.

Theorem 1 Suppose Assumptions B.1, B.2, and B.3 hold. Then,

i. $|\widehat{\beta}-\beta|=o_{p}(1)$ as $j, T \longrightarrow \infty$;

ii. $\sqrt{T}[\widehat{\beta}-\beta] \stackrel{d}{\longrightarrow} \mathcal{N}\left(0, J Z \mathcal{I} Z^{\prime} J^{\prime}\right)$ as $j, T \longrightarrow \infty$ and $\frac{\ln (T)}{j}=o(1)$.

Proof. See Appendix.

The proof proceeds as follows. Item i. in Theorem 1 requires that both $N(\phi)$ and $\widehat{N}_{T}(\phi)$ are $\mathrm{ACMs}$ on $\left(\mathbb{B}, E_{n}\right)$, where $E_{n}$ is the Euclidean metric on $\mathbb{R}^{n}$ and $\mathbb{B}$ is a closed ball centered 
at $\beta$. Lemma 5 gives that $\widehat{N}_{T}(\phi)$ is an ACM on $\left(\mathbb{B}, E_{n}\right)$ and hence $\left|\widehat{N}_{T}(\phi)-\widehat{N}_{T}(\gamma)\right| \leq$ $\kappa|\phi-\gamma|$ holds, with $\gamma, \phi \in \mathbb{B}$ and $\kappa \in[0,1)$. Additionally, the sample mapping has an unique fixed point in $\mathbb{B}$ given by $\widehat{\beta}$, and Lemma 5 establishes that $\widehat{\beta}^{j}$ converges uniformly in $\mathbb{B}$ to $\widehat{\beta}$. It follows that consistency of the IOLS estimator is obtained by using the standard fixed point theorem and Lemma 3 (the sample mapping converges uniformly in probability to its population counterpart). Next, the limiting distribution of $\widehat{\beta}$ also demands $N(\phi)$ and $\widehat{N}_{T}(\phi)$ to be ACMs on $\left(\mathbb{B}, E_{n}\right)$. Furthermore, it requires uniform convergence of $\breve{N}_{T}(\phi)$ to $\widehat{N}_{T}(\phi)$ in $\phi \in \mathbb{B}($ Lemma 2$), \sup _{\phi, \gamma \in \mathbb{B}}\left\|\left[\breve{\Lambda}_{T}(\phi, \gamma)-\Lambda(\phi, \gamma)\right](\phi-\gamma)\right\|=$ $o_{p}(1)$ (Lemma 4), and $\sqrt{T}\left|\widehat{\beta}^{j}-\widehat{\beta}\right|=o_{p}(1)$ as $j, T \longrightarrow \infty$ and $\frac{\ln (T)}{j}=o(1)$ (Lemma 6). It follows that the asymptotic normality result simplifies to the limiting behaviour of $\left[I_{n}-V(\beta)\right]^{-1} \sqrt{T}\left[\breve{N}_{T}(\beta)-\beta\right]$. We follow the work of Francq and Zakoian (1998) and Dufour and Pelletier (2014), and use the central limit theorem of Ibragimov (1962) that encompasses strong mixing processes such as the one in Assumption B.2. In turn, $\sqrt{T}\left[\breve{N}_{T}(\beta)-\beta\right] \stackrel{d}{\longrightarrow} \mathcal{N}\left(0, Z \mathcal{I} Z^{\prime}\right)$ as $j, T \longrightarrow \infty$. This yields an asymptotic variance that is a function of $\mathcal{I}$ rather than the usual $R^{\prime}\left(H \otimes \Sigma_{u}\right) R$ term that appears when $U_{t}$ is an i.i.d. process (see Corollary 1).

Lemma 1 and $\widehat{\beta}$ can be used to compute the empirical counterparts of $V(\beta), H, Z$ and $\Sigma_{u}$, yielding a feasible estimate of the asymptotic variance. Specifically, $\mathcal{I}$ can be consistently estimated by the Newey-West covariance estimator,

$$
\begin{array}{r}
\widehat{\mathcal{I}}=\frac{1}{T-\bar{q}} \sum_{\ell=-m_{T}}^{m_{T}}\left[1-\frac{|\ell|}{m_{T}+1}\right] \sum_{t=\bar{q}+1+|\ell|}^{T}\left\{\left[R^{\prime}\left(\widehat{X}_{t} \otimes I_{K}\right) \widehat{U}_{t}\right] \times\right. \\
\\
\left.\left[R^{\prime}\left(\widehat{X}_{t-\ell} \otimes I_{K}\right) \widehat{U}_{t-\ell}\right]^{\prime}\right\},
\end{array}
$$

where $m_{T}^{4} / T \rightarrow 0$ with $T, m_{T} \rightarrow \infty$.

The practical implication of the violation of item i. in Assumption B.3 is that the IOLS estimator does not converge even for large $T$. If this is the case, the asymptotic results in this section cannot be implemented. By contrast, we note from our simulations that if the IOLS estimator converges, the contraction property assumption is satisfied, i.e. the modulus of the largest eigenvalue of $\widehat{V}_{T}(\widehat{\beta})$ is strictly less than unity. It is also possible to have a DGP satisfying item i. in Assumption B.3 and the IOLS estimator does not converge. This follows because Lemma 5 holds only asymptotically, and convergence in finite sample requires that both the population and sample mappings are ACMs, with the latter holding 
on every iteration. In turn, it is possible to use Lemma 1 and evaluate the modulus of the largest eigenvalue of $\widehat{V}_{T}\left(\widehat{\beta}^{j}\right)$ on every iteration to verify whether the IOLS estimator is in its region of convergence. The Monte Carlo study shows that when the population mapping is an ACM (item i. in Assumption B.3 holds), convergence rates increase monotonically with $T .{ }^{6}$ Additionally, the Monte Carlo section discusses small sample adjustments that improve the convergence rates of the IOLS estimator.

Finally, we derive the consistency and limiting distribution of the IOLS estimator under the stronger assumption that the true data generation process is a strong VARMA process, i.e. $U_{t}$ is an i.i.d. process. The proof works in a similar fashion as the one in Theorem 1. Specifically, all the auxiliary results (Lemmas 2, 3, 4, 5, and 6) hold under the i.i.d. assumption; and the limiting distribution of $\left[I_{n}-V(\beta)\right]^{-1} \sqrt{T}\left[\breve{N}_{T}(\beta)-\beta\right]$ is now derived using the central limit theorem for m.d.s.

Corollary 1 Suppose Assumptions B.1 and B.3 hold. Additionally, assume

i. $U_{t}$ is an i.i.d. process with $\mathbb{E}\left(U_{t}\right)=0, \operatorname{Var}\left(U_{t}\right)=\Sigma_{u}$ and finite fourth moment.

Then, item $i$. in Theorem 1 holds and $\sqrt{T}[\widehat{\beta}-\beta] \stackrel{d}{\longrightarrow} \mathcal{N}\left(0, J Z R^{\prime}\left(H \otimes \Sigma_{u}\right) R Z^{\prime} J^{\prime}\right)$ as $j, T \longrightarrow \infty$ and $\frac{\ln (T)}{j}=o(1)$.

Proof. See Appendix.

\section{Monte Carlo Study}

This section provides results on the finite sample performance of VARMA models estimated with the IOLS methodology. We compare the IOLS estimator with estimators possessing very different asymptotic and computational characteristics. We report results considering five methods: the MLE, the two-stage method (HR) of Hannan and Rissanen (1982), the three-step procedure (HK) of Hannan and Kavalieris (1984), the two-stage method (DJ2) of Dufour and Jouini (2014), and the multivariate version of the three-step procedure (KP) of Koreisha and Pukkila (1990) as discussed in Koreisha and Pukkila (2004) and Kascha (2012). To broadly analyse and assess the performance of the IOLS estimator, we design simulations covering different sample sizes (from 50 to 1,000 observations), system sizes

\footnotetext{
${ }^{6}$ Figure S.1 in the online Supplement displays finite sample convergence rates (heat-map) of the IOLS estimator for $\operatorname{ARMA}(1,1)$ models defined as $y_{t}=\beta_{1} y_{t-1}+u_{t}+\beta_{2} u_{t-1}$. We show that convergence rates improve dramatically when sample size increases from $T=100$ to $T=10,000$ in the entire set of parameters satisfying item i. in Assumption B.3.
} 
$(K=3, K=10, K=20, K=40$, and $K=52)$, Kronecker indices, dependencies among the variables, and both weak and strong processes. ${ }^{7}$

We simulate stable, invertible, and unique $\operatorname{VARMA}(1,1)$ models as

$A_{0} Y_{t}=A_{1} Y_{t-1}+A_{0} U_{t}+M_{1} U_{t-1}$.

Uniqueness is imposed through the Echelon form transformation, which implies $A_{0}=M_{0}$ in (19) and requires a choice of Kronecker indices. We discuss results considering six DGPs. DGPs I and II set all Kronecker indices to one, which implies that $A_{0}=I_{K}$ and $A_{1}$ and $M_{1}$ are full matrices. These DGPs differ with respect to the eigenvalues assigned to the parameter matrices. The eigenvalues of $A_{1}$ and $M_{1}$ in DGP I are constant and equal to 0.5, whereas the eigenvalues in DGP II take positive, negative and near-to-zero values. Precisely, the eigenvalues of $A_{1}$ and $M_{1}$ are $(0.80,0.20,0.05)^{\prime}$ and $(0.90,0.02,0.20)^{\prime}$, respectively. ${ }^{8}$ For DGPs III, IV, V, and VI, the first $k$ Kronecker indices are set to one, while the remaining $K-k$ Kronecker indices are set to zero, so that $\mathbf{p}=\left(p_{1}, p_{2}, \ldots, p_{K}\right)^{\prime}$ with $p_{i}=1$ for $i \leq k$ and $p_{i}=0$ for all $i>k$. Specifically, DGP III has $k=1$, while DGPs IV, V and VI have $k=2, k=3$, and $k=6$, respectively. The free parameters in DGPs III, IV, V, and VI are based on real data and chosen as the estimates obtained by fitting VARMA(1,1) models to the datasets in Section 5. DGPs III-VI are particularly relevant because they reduce dramatically the number of free parameters in (19), while yielding rich dynamics in the MA component of the standard representation of (19). ${ }^{9}$

Weak VARMA $(1,1)$ models are obtained by generating $U_{t}$ as in Romano and Thombs, 1996, p. 591, with $U_{t}=\prod_{\ell=0}^{m} \varepsilon_{t-\ell}$, where $m=3$ and $\varepsilon_{t}$ is a zero mean i.i.d. process with covariance matrix $I_{K}$. This procedure yields uncorrelated innovations satisfying the mixing conditions stated in Assumption B.2. We summarize results for each specification using two measures: MRRMSE and Share. MRRMSE accounts for the mean of the relative root median squared error (RRMSE) measures of all parameters, where RRMSE is the ratio of the root median squared error (RMSE) obtained from a given estimator over the RMSE of the HR estimator. RRMSE measures lower than one indicate that the HR estimator is outperformed by the alternative estimator. Share is the frequency a given estimator returns

\footnotetext{
${ }^{7}$ For sake of brevity, results for the strong VARMA specifications are in the online Supplement.

${ }^{8}$ File "DGPsfile.csv" (available online) contains the true values used in all simulations in this section.

${ }^{9}$ Because DGPs III-VI imply that $A_{0}$ is an invertible lower triangular matrix, multiplying (19) by $A_{0}^{-1}$ yields its standard representation, $Y_{t}=A_{1}^{*} Y_{t-1}+U_{t}+M_{1}^{*} U_{t-1}$, where $A_{1}^{*}:=A_{0}^{-1} M_{1}=\left(A_{1, K \times k}^{*}, 0\right)$ and $M_{1}^{*}:=A_{0}^{-1} M_{1}$ is a full matrix.
} 
the lowest RRMSE over all the free parameters. ${ }^{10}$ The MRRMSE and Share measures are only computed using the replications that achieved convergence and satisfy Assumption B.1. ${ }^{11}$ We discard the initial 500 observations and fix the number of replications to 5,000, unless otherwise stated. We report only a fraction of the entire set the Monte Carlo results. The complete set of tables is in Section S.3 in the online Supplement.

A valid concern that emerges when estimating DGPs I and II with the IOLS estimator is its rather low convergence rates for small sample sizes. Nevertheless, convergence rates of the IOLS estimator increase monotonically with $T$ in both DGPs and system dimensions ( $K=3$ and $K=10$ ), which leave us with the conclusion that this is a small sample issue. This is in line with our theoretical results, as Lemma 1 holds when evaluated at the true vector of parameters and Lemma 5 holds only asymptotically. We address the causes for the IOLS estimator failing to achieve convergence and identify three reasons for this finite sample anomaly. The IOLS fails when, first, it generates at some iteration a non-invertible VARMA model that contains no roots on the unit circle; second, it generates a non-stable VARMA model with no roots on the unit circle; third, it converges to multiple (finite) fixed points. We address these issues individually in the IOLS estimator. Namely, first, in the case of a non-invertible VARMA model, we convert the non-invertible moving average polynomial to its corresponding invertible representation using Lippi and Reichlin's 1994 procedure and continue iterating. Second, in the instance of a non-stable VARMA model, we factorize the VAR polynomial and replace the eigenvalues greater than one in absolute value by 0.99 and continue iterating. Finally, in the case of convergence to multiple fixed points, we choose the one which minimizes the determinant of the covariance matrix of the residuals and stop iterating. ${ }^{12}$ Implementing these small sample adjustments improves the convergence rates and, most importantly, does not come at expense of worse results. ${ }^{13}$ In short, considering DGPs I and II with sample sizes of $T \leq 400$, convergence rates increase an average of $34 \%$ and $56 \%$ for $K=3$ and $K=10$, respectively, while MRRMSE measures increase by only $3 \%$ and $1 \%$ for $K=3$ and $K=10$, respectively. Numbers are even more

\footnotetext{
${ }^{10}$ As an example, when $K=3$ and $\mathbf{p}=(1,0,0)^{\prime}, n=6$, i.e. there are six free parameters (excluding the distinct covariance parameters) to be estimated. If the IOLS estimator has a Share of $67 \%$, it implies that the IOLS estimator delivers the lowest RRMSE in four out of those six free parameters. This measure is particularly informative when dealing with systems with large number of free parameters.

${ }^{11}$ Throughout this section, we assume an estimator converges if its final estimates satisfy Assumption B.1. For the IOLS and MLE estimators, convergence also implies numerical convergence of their respective algorithms.

${ }^{12}$ Section S.3.2 details the implementation of the small sample adjustments and discusses their finite sample performance.

${ }^{13}$ See Tables S.6-S.13 in the online Supplement.
} 
favourable when considering DGP II, as convergence rates increase by $59 \%$ and $89 \%$ for $K=3$ and $K=10$, respectively, while MRRMSE measures increase by only $5 \%$ and $2 \%$ for $K=3$ and $K=10$, respectively. Despite the improvements in the convergence rates, we take a conservative stand and report only results for the IOLS estimator computed without any finite sample adjustments (Tables 1 and 2). When appropriate, we further discuss the convergence rates and performances of the small sample adjustments.

The first set of Monte Carlo simulations addresses the finite sample performance of the IOLS estimator in small sized $(K=3) \operatorname{VARMA}(1,1)$ models. We simulate weak VARMA(1,1) models considering DGPs I, II and III. DGPs I and II yield 18 free parameters in the $A_{0}, A_{1}$, and $M_{1}$ parameter matrices, while DGP III has 6 free parameters in these matrices. We consider samples of 50, 100, 150, 200, and 400 observations. Table 1 presents the results. First, we find that the MLE estimator is dominant in DGP I. This is not surprising, because MLE is known to perform well on specifications where the absolute eigenvalues are bounded away from zero and one (Kascha (2012)). The DGP II specification is numerically more difficult to handle, yielding lower rates of convergence for both the IOLS and MLE estimators. Nevertheless, the IOLS estimator is the one which delivers the best results considering both MRRMSE and Share measures. This indicates that if convergence is achieved, the IOLS estimator is able to handle systems with near-to-zero eigenvalues more efficiently than the benchmark MLE estimator. Furthermore, when considering the IOLS computed with the small sample adjustments discussed before, convergence rates for the DGP II increase to $53 \%, 67 \%, 72 \%, 75 \%$, and $82 \%$ for $T=50, T=100, T=150, T=200$, and $T=400$, respectively. The IOLS estimator is very competitive in DGP III and presents the highest Share measure in all sample sizes. Simulating DGPs I, II, and III for $K=10$ provides a comparable picture (see detailed discussion in the online Supplement).

Table 2 displays results covering the finite sample performance of the IOLS estimator in medium and large sized systems. We simulate DGPs III, IV, V, and VI for $K \in$ $(10,20,40,52)$, which mimic our empirical study. To the best of our knowledge, this is the first study to consider such high-dimensional VARMA models in a Monte Carlo study. The sample size and number of replications are set to $T=400$ and 1,000, respectively. These are high-dimensional models with the number of free parameters (excluding the distinct covariance parameters), $n$, varying from 20 to 624 . We find that the IOLS estimator presents the best relative performance (in terms of both the MRRMSE and Share measures) for 
large systems $(K=40$ and $K=52)$. These findings hold for all DGPs. The relative performance of the HK and IOLS estimators are outstanding, with an average improvement with respect to the HR estimator of up to $65 \%$. On average, the IOLS outperforms the HK estimator in 15\% (in terms of the MRRMSE measure). Considering systems with $K=10$ and $K=20$, the HK estimator is the one that delivers the best performance for most specifications, while the IOLS estimator is constantly ranked as the second best estimator. $^{14}$ Therefore, the IOLS estimator considerably improves its performance when estimating high-dimensional restricted models with $A_{0} \neq I_{K}$, while remaining a feasible alternative (average convergence rate of 95\%). These results motivate the use of the Echelon form transformation in the fashion of DGPs III-VI and the IOLS estimator as feasible alternative to high-dimensional VARMA $(1,1)$ models.

Overall, we conclude that the IOLS estimator is a competitive alternative and compares favourable with its competitors in a variety of cases: small sample sizes, small sized systems with near-to-zero eigenvalues, and large sized systems with many Kronecker indices set to zero. The MLE and HK estimators also present remarkable performances in terms of RMSE, which is in line with previous studies (Kascha (2012)).

\section{Empirical Application}

In this section, we analyse the competitiveness of VARMA models estimated with the IOLS procedure to forecast macroeconomic variables. We forecast three key macroeconomic variables: industrial production (IPS10), interest rate (FYFF), and CPI inflation (PUNEW). We assess VARMA forecast performance under different system dimensions and forecast horizons.

\subsection{Data and Setup}

We use US monthly seasonally adjusted data from the Stock and Watson (2006) dataset, which runs from 1959:1 through 2003:12. The choice of using seasonally adjusted data is in line with the large datasets literature (see Stock and Watson (2002a,b), De Mol, Giannone, and Reichlin (2008), and Bańbura, Giannone, and Reichlin (2010)), as seasonal data intensifies the "curse of dimensionality" by requiring the inclusion of extra lags. Therefore,

\footnotetext{
${ }^{14}$ The online Supplement presents results where IOLS outperforms the HK estimator for $K=10$ and $K=20$ in DGPs IV-VI with $T=200$.
} 
as usual in this literature, a practitioner should be cautious when using the framework discussed in this section for seasonal data. As in Carriero, Kapetanios, and Marcellino (2011), we use 52 macroeconomic variables that represent the main categories of economic indicators. We work with five system dimensions: $K \in(3,10,20,40,52)$. We construct four different datasets (one to four) for each system size where $10 \leq K \leq 40$, as a way to assess robustness of the VARMA framework when dealing with different explanatory variables. When selecting the variables, we try to keep a balance among the three main categories of data: real economy, money and prices, and financial market. ${ }^{15}$ The series are transformed, as in Carriero, Kapetanios, and Marcellino (2011), in such a way that they are approximately stationary. The forecasting exercise is performed in pseudo real time, with a fixed rolling window of 400 observations. All models considered in the exercise are estimated in every window. We perform 115 out-of-sample forecasts considering six different horizons: one- (Hor:1), two- (Hor:2), three- (Hor:3), six- (Hor:6), nine- (Hor:9) and twelve- (Hor:12) steps-ahead.

We compare the different VARMA specifications with five alternative methods: AR(1), $\operatorname{ARMA}(1,1), \operatorname{VAR}\left(p^{*}\right)$, BVAR, and factor models. ${ }^{16} \operatorname{ARMA}(1,1)$ models are estimated using the IOLS and maximum-likelihood estimators. We estimate the BVAR model with the normal-inverted Wishart prior as in Bańbura, Giannone, and Reichlin (2010), so that the prior is adjusted to accommodate the fact that our variables are approximately stationary. ${ }^{17}$ We report results considering three BVAR specifications. The first specification, BVAR ${ }_{\mathrm{SC}}$, is obtained by setting the hyperparameter $\varphi$ (tightness parameter) to the value which minimizes the SC criterion over a grid of $\varphi \in(2.0 e-5,0.0005,0.002,0.008,0.018,0.072,0.2$, $1,500) .{ }^{18}$ The second specification, $\operatorname{BVAR}_{0.2}$, sets $\varphi=0.2$, which is the default choice in the package Regression Analysis of Time Series (RATS) and it is the benchmark model in Carriero, Kapetanios, and Marcellino (2011). The third specification, BVAR opt $_{\text {, follows from }}$ Bańbura, Giannone, and Reichlin (2010) and chooses $\varphi \in(2.0 e-5,0.0005,0.002,0.008$, $0.018,0.072,0.2,1,500)$ which minimizes the in-sample one-step-ahead root mean squared forecast error in the last 24 months of the sample. For the three different specifications, we

\footnotetext{
${ }^{15}$ Table S.14 in the online Supplement reports the details of the different datasets.

${ }^{16}$ The lag length $p^{*}$ in the $\operatorname{VAR}\left(p^{*}\right)$ specification is obtained by minimizing the AIC criterion.

${ }^{17}$ See the online Supplement (Section S.4.1) for an extended discussion on the BVAR framework implemented in this section.

${ }^{18}$ This grid follows Carriero, Kapetanios, and Marcellino (2011) and it is broad enough to include both very tight $(\varphi=2.0 e-5)$ and loose $(\varphi=500)$ hyperparameters.
} 
choose the lag length that minimizes the SC criterion. ${ }^{19}$ We grid search over $\varphi$ and the optimal lag length on every rolling window. Factor models summarize a large number of predictors in only a few number of factors. We adopt the two-step procedure as in Stock and Watson $(2002 \mathrm{a}, \mathrm{b})$. In the first step, factors $\left(\left\{F_{t}\right\}_{t=1}^{T}\right)$ are extracted via principal components, whereas the second step consists of projecting $y_{i, t+h}$ onto $\left(\widehat{F}_{t}^{\prime}, \ldots, \widehat{F}_{t-\ell}^{\prime}, y_{i, t-1}, \ldots, y_{i, t-r}\right)$, with $\ell \geq 0$ and $r>0$. We determine the lag orders by minimizing the Schwarz (SC) criterion, and choose the number of factors according to the $\mathrm{SC}$ and $I C_{p 3}$ criteria, denoted as $F M_{S C}$ and $F M_{I C 3}$, respectively (Stock and Watson (2002a)). Factors are computed using only the variables available on the respective dataset.

When dealing with empirical data, the Kronecker indices which are required to express a VARMA representation in its Echelon form are unknown. We determine them using three strategies. The first one imposes rank reduction on the parameter matrices of the VARMA(1,1) model in a similar fashion as the DGPs III, IV, V, and VI in the Monte Carlo section. This follows Carriero, Kapetanios, and Marcellino (2011), who show that reduced rank VAR (RRVAR) models perform well when forecasting macroeconomic variables using large datasets. By specifying the Kronecker indices as $\mathbf{p}=\left(p_{1}, p_{1}, \ldots, p_{K}\right)^{\prime}$ with $p_{i}=1$ for $i \leq k$ and $p_{i}=0$ for all $i>k$, the rank of both parameter matrices of the standard VARMA representation $A_{1}^{*}=A_{0}^{-1} A_{1}$ and $M_{1}^{*}=A_{0}^{-1} M_{1}$ reduces to $k$, in that the $\operatorname{VAR}(\infty)$ representation of the $\operatorname{VARMA}(1,1)$ model is a $\operatorname{RRVAR}(\infty)$ of rank $k$. We report results for $k \in(1,2,3,4,5,6)$ denoted as $\mathbf{p}_{k=1}, \mathbf{p}_{k=2}, \mathbf{p}_{k=3}, \mathbf{p}_{k=4}, \mathbf{p}_{k=5}$, and $\mathbf{p}_{k=6}$. Notably, the restrictions and number of free parameters in $\mathbf{p}_{k=1}, \mathbf{p}_{k=2}, \mathbf{p}_{k=3}$, and $\mathbf{p}_{k=6}$ are analogous to DGPs III, IV, V, and VI, respectively. The second and third strategies estimate the Kronecker indices directly from the data. Specifically, the second strategy adopts the Hannan-Kavalieris algorithm (denoted as $\mathbf{p}_{H K}$ ), which consists of choosing Kronecker indices that minimize the $\mathrm{SC}_{K}$ criterion (Lütkepohl, 2007, p. 503). ${ }^{20}$ We implement this procedure on every rolling window for $K=3$ and $K=10$, and on the first rolling window for medium and large sized datasets $(K=20, K=40$, and $K=52)$. We then carry the estimated Kronecker indices to the subsequent rolling windows. The maximum value of the Kronecker indices is set to one.

The third strategy is based on Poskitt (1992) and reviewed in Lütkepohl and Poskitt

\footnotetext{
${ }^{19}$ The maximum lag length is set to be 15,8 , and 6 for $K \leq 10,20 \leq K \leq 40$, and $K=52$, respectively.

${ }^{20}$ See Section S.4.2 in the online Supplement for a complete description of the Hannan-Kavalieris procedure.
} 
(1996). We refer to this method as the Lütkepohl-Poskitt procedure and denote it as $\mathbf{p}_{L P} \cdot{ }^{21}$ The Lütkepohl-Poskitt procedure is based on the property of the Echelon form that the restrictions on the $k^{\text {th }}$ equation do not depend on the Kronecker indices $p_{i}>p_{k}$. The Lütkepohl-Poskitt procedure consists of estimating each of the $K$ equations in the system by least squares (using the residuals from $\operatorname{VAR}(\tilde{p})$ instead of the true disturbances) and evaluating model selection criteria for each of the $K$ equations separately. ${ }^{22}$ Because the Kronecker indices are estimated equation by equation, the Lütkepohl-Poskitt searches for the first local minimum of the criterion for each equation, and hence it suits particularly well high-dimensional models. The Lütkepohl-Poskitt procedure is consistent under suitable conditions (Poskitt (1992)). Specifically, if Assumptions B.1 and B.3 hold and $U_{t}$ is an i.i.d. process, choosing $\tilde{p}$ with the AIC criterion and setting the model selection criterion as the SC criterion meet these conditions. The Lütkepohl-Poskitt procedure has two important advantages compared to the Hannan-Kavalieris algorithm. First, it does not impose a prespecified upper bound to the Kronecker indices. This implies that very general and highly complex VARMA(p,q) models with $p, q \geq 1$ and up to $K$ nonzero Kronecker indices can be selected. Despite its general approach, the Lütkepohl-Poskitt procedure also attains some degree of sparseness and parsimony, as it penalizes for the number of freely varying parameters in each equation. Second, the Lütkepohl-Poskitt procedure is preferable from a computational point of view, as it allows the estimation of the Kronecker indices on every rolling window for all system sizes. To assess how these different strategies fit the data, we report two $\mathrm{SC}$ criteria, denoted as $\mathrm{SC}_{K}$ and $\mathrm{SC}_{3} . \mathrm{SC}_{K}$ is the standard $\mathrm{SC}$ criterion computed with the entire $(K \times K)$ covariance matrix of the residuals, whereas $\mathrm{SC}_{3}$ uses only the $(3 \times 3)$ upper block of the residuals covariance matrix, as this contains the covariance matrix of the three key macroeconomic variables. The $\mathrm{SC}_{3}$ criterion, therefore, is a measure of fit that is solely related to the variables that we are ultimately interested in. All VARMA specifications are estimated with the IOLS estimator discussed in Section $2 .^{23}$

We compare different models using the out-of-sample relative mean squared forecast error (RelMSFE) computed using the AR(1) as a benchmark. We choose this benchmark, because it makes easy the comparison across the different datasets and system dimensions. We assess the predictive accuracy with the Diebold and Mariano (1995) test. The use of

\footnotetext{
${ }^{21}$ We thank an anonymous referee for suggesting this procedure.

${ }^{22}$ See Section S.4.3 in the online Supplement for a complete description of the Lütkepohl-Poskitt procedure.

${ }^{23}$ If the IOLS does not converge, we implement the small sample adjustments discussed in Section 4 . If convergence is not achieved, we adopt the consistent initial estimates: the two-stage HR estimator.
} 
this test is justified given our focus on forecasts obtained through rolling windows.

\subsection{Results}

We organize the results as follows. Table 3 reports a summary of the forecast results across the different datasets and presents, for each system size, three panels. The first panel reports the frequency (in percentage points) for which at least one of the VARMA specifications $\left(\mathbf{p}_{k=1}, \mathbf{p}_{k=2}, \mathbf{p}_{k=3}, \mathbf{p}_{k=4}, \mathbf{p}_{k=5}, \mathbf{p}_{k=6}, \mathbf{p}_{H K}\right.$, and $\mathbf{p}_{L P}$ ) outperforms (delivers the lowest RelMSFE measures) the assigned group of competitors in a given forecast horizon. ${ }^{24}$ Similarly, the second and third panel display the frequencies for which the most restricted, $\mathbf{p}_{k=1}$, and general, $\mathbf{p}_{L P}$, specifications outperform the assigned group of competitors. We consider five groups of competitors: AR, ARMA, VAR, FM, and BVAR. The AR group collects the AR(1) specification; ARMA has the ARMA(1,1) specification estimated with IOLS and MLE; VAR contains the $\operatorname{VAR}\left(p^{*}\right)$ model; FM gathers the different factor model specifications, namely the $F M_{S C}$ and $F M_{I C 3}$; and the BVAR collects the three BVAR specifications: $\mathrm{BVAR}_{\mathrm{SC}}, \mathrm{BVAR}_{0.2}$ and $\mathrm{BVAR}_{\text {opt }}$. Finally, Table 4 compares the performance of the IOLS estimator with the DJ2, HK, and KP estimators. We report the frequency (in percentage points) for which the IOLS estimator outperforms the alternative estimators for each forecast horizon. A comprehensive set of results is available in Section S.4.4 in the online Supplement (Tables S.15 to S.28), making it possible to assess the forecast performance of all VARMA specifications and the alternative model competitors in all datasets and system sizes up to the level of the key macroeconomic variables. Convergence rates for the IOLS estimator are also reported for all VARMA specifications. In what follows, we draw from these tables when discussing the empirical results.

Starting with $K=3$, we find that VARMA models largely outperform the AR, VAR, FM, and the BVAR groups up to the sixth-step-ahead forecast (see Table 3). Specifically, the $\mathbf{p}_{k=1}$ and $\mathbf{p}_{k=2}$ specifications are the ones which deliver the best results. VARMA models also outperform the $\operatorname{ARMA}(1,1)$ specifications, which reinforces the idea that modelling the three key macroeconomic variables in a multivariate context pays off. Taking into account all forecast horizons, VARMA models deliver the best forecast in $67 \%$ of the cases.

We now summarize the results for the medium and large datasets, $(K=10, K=20$, $K=40$, and $K=52$ ). We start by discussing the results for $K=10$. For short horizons

\footnotetext{
${ }^{24}$ Percentages are computed across the three key macroeconomic variables and the four datasets discussed in Section 5.1.
} 
(Hor:1 - Hor:6), VARMA models deliver the lowest RelMSFE in 63\% of the cases. Compared with the BVAR group, VARMA models remain dominant, delivering more accurate forecasts in $81 \%$ of the cases (Hor:1 - Hor:6). Moreover, the $\mathbf{p}_{k=1}$ and $\mathbf{p}_{k=2}$ specifications are the ones that usually deliver the lowest RelMSFE measures among the VARMA specifications. Specifically, $\mathbf{p}_{k=1}$ minimizes the $\mathrm{SC}_{3}$ criterion in all datasets, which indicates that choosing the Kronecker indices that minimize the $\mathrm{SC}_{3}$ criterion pays off in terms of forecast accuracy. Increasing the number of nonzero Kronecker indices from three to six, $\mathbf{p}_{k=3}, \mathbf{p}_{k=4}$, $\mathbf{p}_{k=5}$, and $\mathbf{p}_{k=6}$ specifications, delivers stable RelMSFE measures which are less often the best among the competitors. This is in line with the large datasets literature, which documents that imposing restrictions on the parameter matrices of a standard VAR model improves forecast accuracy (De Mol, Giannone, and Reichlin (2008), Carriero, Kapetanios, and Marcellino (2011), and Bańbura, Giannone, and Reichlin (2010)). Choosing the Kronecker indices according to the general Lütkepohl-Poskitt procedure and the Hannan-Kavalieris algorithm typically yields more complex models (up to eight nonzero Kronecker indices), stable RelMSFE measures, and a slightly less accurate forecast performance. Specifically, while the $\mathbf{p}_{L P}$ generally outperforms the AR, ARMA, and VAR for short horizons, it is outperformed by FM, and BVAR specifications. With regard to the estimation of VARMA models, the IOLS estimator works well, presenting an average convergence rate of $93 \%$. Additionally, its relative performance with respect to the DJ2, HK, and KP estimators is positive. Considering the $\mathbf{p}_{k=1}$ and the $\mathbf{p}_{k=2}$ specifications, the IOLS estimator outperforms its linear competitors in $81 \%$ of the cases (Table 4 ).

We now discuss the results for $K=20$. Overall, VARMA models are very competitive in the short horizons (Hor:1 - Hor:6), outperforming the AR, ARMA, VAR, FM and BVAR groups in $90 \%, 71 \%, 94 \%, 79 \%$, and $88 \%$ of the cases, respectively. Results are also stable across the different datasets and VARMA specifications, showing robustness of the VARMA framework. Differently from the $K=10$ scenario, there is not a clear winner among the VARMA specifications, although $\mathbf{p}_{k=1}$ remains very competitive and minimizes the $\mathrm{SC}_{3}$ criterion. While setting the number of nonzero Kronecker indices as $k \in(2,3,4,5,6)$ typically does not improve forecast accuracy ( $\mathbf{p}_{k=6}$ in Dataset 3 is the exception), the data driven Lütkepohl-Poskitt procedure emerges as a strong competitor and yields the most accurate forecasts among all VARMA specifications in $29 \%$ of the cases. The IOLS estimator presents rates of convergence averaging 94\% (across all specifications) and usually delivers 
more accurate forecasts than the DJ2, HK, and KP estimators in the long horizons. The strong performance of the HK estimator in the short horizons is in line with the Monte Carlo results.

Considering the case of large datasets $(K=40)$, we find that VARMA models stay very competitive, outperforming the AR, ARMA, VAR, FM, and BVAR groups in 92\%, 81\%, $96 \%, 58 \%$, and $82 \%$ of the cases, respectively. The general $\mathbf{p}_{L P}$ specification now emerges as the clear winner among the VARMA specifications, as it produces the most accurate forecasts in $46 \%$ of the cases. In turn, the Lütkepohl-Poskitt procedure is able to select the relevant Kronecker indices to forecast the key macroeconomic variables and preserves some degree of sparseness in the parameter matrices, which is important when forecasting using rich datasets. The good performance of the $\mathbf{p}_{L P}$ specification is also due to the use of the IOLS estimator, as the IOLS estimator outperforms the DJ2, HK, and KP estimators in $68 \%, 71 \%$, and $94 \%$ of the cases (Table 4). Indeed, the Monte Carlo simulations show that the IOLS estimator delivers an outstanding performance in large sized systems, $(K=40$ and $K=52$ ). We report marginal gains in terms of forecast accuracy when moving from the $\mathbf{p}_{k=1}$ specification to the more general models where the number of nonzero Kronecker indices are $k \in(2,3,4,5,6)$. Similarly to the case of the $\mathbf{p}_{L P}$ specification, the IOLS estimator systematically delivers more accurate forecasts than the alternative estimators. Considering the specifications with $k \in(1,2,3,4,5,6)$, the IOLS estimator outperforms the DJ2, HK, and KP estimators in $79 \%, 87 \%$, and $86 \%$ of the cases, respectively (Table 4), and improves the RelMSFE measures in 19\%, 33\%, and $40 \%$ on average for the DJ2, HK, and KP estimators, respectively (Hor:1 - Hor:6). ${ }^{25}$ Finally, the IOLS estimator remains a robust alternative, achieving convergence in $93 \%$ of the rolling windows (all specifications).

We now turn our attention to the results using the entire dataset $(K=52)$. When comparing the performance of VARMA models with the BVAR group, the former delivers lower RelMSFE measures in $72 \%$ of the cases. This is a strong result in favour of VARMA models, since BVAR specifications are known to be very competitive when forecasting with large datasets. Overall, factor models deliver the best performance. Among the VARMA specifications, $\mathbf{p}_{k=1}$ and $\mathbf{p}_{k=2}$ deliver the best results. Increasing the number of nonzero Kronecker indices to $k \in(3,4,5,6)$ delivers at most marginal improvements in terms of RelMSFE measures. When the IOLS estimator presents low convergence rates $\left(\mathbf{p}_{L P}\right.$ and

\footnotetext{
${ }^{25}$ Results available upon request.
} 
$\mathbf{p}_{H K}$ specifications), VARMA models become less competitive. Alternatively, using the RelMSFE measures obtained with the DJ2, HK, and KP estimators does not help either, as they yield no qualitative improvement in terms of forecast hierarchy when compared to the IOLS based measures. On the comparison of the IOLS and the alternative linear estimators, the IOLS estimator largely outperforms (presents values greater than 50\%) the DJ2, HK, and KP estimators for the $\mathbf{p}_{k=1}, \mathbf{p}_{k=2}, \mathbf{p}_{k=3}, \mathbf{p}_{k=4}, \mathbf{p}_{k=5}$, and $\mathbf{p}_{k=6}$ specifications. Specifically, the IOLS estimator outperforms the DJ2, HK, and KP estimators in 83\%, 98\%, and $94 \%$ of the cases, respectively (Table 4), and improves the RelMSFE measures compared to the DJ2, HK, and KP estimators an average of 33\%, 54\%, and 70\%, respectively, (Hor:1 Hor:3). ${ }^{26}$ Finally, convergence rates for these specifications are $100 \%$.

To sum up the results of this section, VARMA models estimated using the IOLS estimator are generally very competitive and able to beat the four most prominent competitors in this type of study: AR, ARMA, factor models and BVAR models. This finding is especially present for the $\mathbf{p}_{k=1}$ and $\mathbf{p}_{L P}$ specifications. VARMA results are also stable across the different datasets and Kronecker indices specifications, indicating that the framework adopted is fairly robust. Considering all system sizes and datasets, VARMA specifications deliver the lowest RelMSFE measures for the one-, two-, three-, and six-month-ahead forecast in $63 \%$ of the cases, indicating that VARMA models are indeed strong candidates to forecast key macroeconomic variables using small, medium and large sized datasets. It is particularly relevant to highlight the performance of VARMA models relative to the BVAR models, as VARMA models systematically deliver more accurate forecasts for all datasets. The IOLS estimator is a valid alternative to deal with large and complex VARMA systems (convergence rates averaging $92 \%$ ) and compares favourably with the main linear competitors (more accurate forecasts in $69 \%$ of the cases). Finally, our findings reinforce two important aspects: using the Echelon form transformation either in the fashion of DGPs III-VI or using the Lütkepohl-Poskitt procedure is a powerful tool to deal with high-dimensional models; and that IOLS estimator is particularly suitable to estimate high-dimensional VARMA models, as the Monte Carlo simulations and empirical results suggest.

\footnotetext{
${ }^{26}$ Results available upon request.
} 


\section{Conclusion}

This paper addresses the issue of modelling and forecasting key macroeconomic variables using rich (small, medium and large sized) datasets. We propose the use of VARMA models as a feasible framework for this task. We overcome the natural difficulties in estimating medium- and high-dimensional VARMA models with the MLE framework by adopting the IOLS estimator.

We establish the consistency and asymptotic distribution for the IOLS estimator by considering the general weak and strong $\operatorname{VARMA}(\mathrm{p}, \mathrm{q})$ models. It is also important to point out that our theoretical results are obtained under weak assumptions that are compatible with the quasi-maximum-likelihood (QMLE) estimator. The extensive Monte Carlo study shows that the IOLS estimator is feasible and consistent in small and high-dimensional systems. Furthermore, the IOLS estimator outperforms the MLE and other linear estimators, in terms of mean squared error, in a variety of scenarios: when $T$ is small; disturbances are weak; near-to-zero eigenvalues; and high-dimensional models $(K=40$ and $K=52)$. The empirical results show that VARMA models perform better than $\operatorname{AR}(1), \operatorname{ARMA}(1,1)$, VAR, BVAR, and factor models for different system sizes and datasets. We find that VARMA models estimated with the IOLS estimator are very competitive at forecasting short horizons (one-, two-, three- and six-month-ahead horizons) in small, medium, and large sized datasets. In particular, the $\mathbf{p}_{k=1}$ and the general $\mathbf{p}_{L P}$ specifications most often emerge as the ones which produce the most accurate results among all VARMA specifications. Finally, we find that VARMA models estimated with the IOLS estimator usually deliver better forecasts than the ones estimated with the alternative linear estimators.

\section{References}

AkaIke, H. (1974): "A New Look at the Statistical Model Identification," IEEE Transactions on Automatic Control, 19, 667-674.

(1976): "Canonical Correlation Analysis of Time Series and the Use of an Information Criterion," in System Identification, ed. by L. D. Mehar, R., pp. 27-96. New York: Academic Press.

Athanasopoulos, G., D. S. Poskitt, and F. Vahid (2012): "Two Canonical VARMA 
Forms: Scalar Component Models Vis-à-Vis the Echelon Form," Econometric Reviews, $31(1), 60-83$.

Athanasopoulos, G., and F. Vahid (2008): "A Complete VARMA Modelling Methodology Based on Scalar Components," Journal of Time Series Analysis, 29, 533-554.

Bańbura, M., D. Giannone, and L. Reichlin (2010): "Large Bayesian vector auto regressions," Journal of Applied Econometrics, 25, 71-92.

Bernanke, B., J. Boivin, And P. S. Eliasz (2005): "Measuring the Effects of Monetary Policy: A Factor-augmented Vector Autoregressive (FAVAR) Approach," The Quarterly Journal of Economics, 120(1), 387-422.

Blanchard, O., And D. Quah (1989): "The dynamic effects of aggregate demand and supply disturbances," American Economic Review, 79, 655-673.

Carriero, A., G. Kapetanios, and M. Marcellino (2011): "Forecasting large datasets with Bayesian reduced rank multivariate models," Journal of Applied Econometrics, 26, $735-761$

Chan, J. C., E. Eisenstat, and G. Koop (2016): "Large Bayesian VARMAs," Journal of Econometrics, 192(2), 374 - 390, Innovations in Multiple Time Series Analysis.

De Mol, C., D. Giannone, and L. Reichlin (2008): "Forecasting Using a Large Number of Predictors: Is Bayesian Regression a Valid Alternative to Principal Components?," Journal of Econometrics, 146, 318-328.

Diebold, F. X., And R. S. Mariano (1995): "Comparing Predictive Accuracy," Journal of Business \& Economic Statistics, 13, 253-263.

Dominitz, J., and R. Sherman (2005): "Some Convergence Theory for Iterative Estimation Procedures with an Application to Semiparametric Estimation," Econometric Theory, 21, 838-863.

Dufour, J.-M., And T. Jouini (2005): "Asymptotic Distribution of a Simple Linear Estimator for Varma Models in Echelon Form," in Statistical Modeling and Analysis for Complex Data Problems, ed. by P. Duchesne, and B. Rémillard, pp. 209-240. Springer US. 
(2014): "Asymptotic Distributions for Quasi-Efficient Estimators in Echelon VARMA Models," Computational Statistics $\&$ Data Analysis, 73, 69-86.

Dufour, J.-M., And D. Pelletier (2014): "Practical Methods for Modelling Weak VARMA Processes: Identification, Estimation and Specification with a Macroeconomic Application," Manuscript.

Dufour, J.-M., And D. Stevanović (2013): "Factor-Augmented VARMA Models With Macroeconomic Applications," Journal of Business 63 Economic Statistics, 4, 491-506.

Forni, M., M. Hallin, M. Lippi, and L. Reichlin (2000): "The Generalised Dynamic Factor Model: Identification and Estimation," Review of Econometrics and Statistics, $82(4), 540-554$.

FrancQ, C., R. Roy, And J.-M. Zakoian (2005): "Diagnostic Checking in ARMA Models with Uncorrelated Errors," Journal of the American Statistical Association, 100, $532-544$.

FrancQ, C., AND J.-M. ZAKOIAN (1998): "Estimating Linear Representations of Nonlinear Processes," Journal of Statistical Planning and Inference, 68, 145-165.

(2005): "Recent Results for Linear Time Series Models with Non Independent Innovations," in Statistical Modeling and Analysis for Complex Data Problems, ed. by P. Duchesne, and B. RMillard, pp. 241-265. Springer US.

Gourieroux, C., And A. Monfort (2015): "Revisiting Identification and Estimation in Structural VARMA Models," Discussion paper, CREST.

Hannan, E. J. (1969): “The Identification of Vector Mixed Autoregressive-Moving Average Systems," Biometrika, 56(1), 223-225.

_ (1976): "The Identification and Parameterization of Armax and State Space Forms," Econometrica, 44(4), 713-723.

Hannan, E. J., And M. Deistler (1988): The Statistical Theory of Linear Systems. John Wiley and Sons, New York.

Hannan, E. J., and L. Kavalieris (1984): "Multivariate Linear Time Series Models," Advances in Applied Probability, 16(3), 492-561. 
Hannan, E. J., And A. J. McDougall (1988): "Regression Procedures for ARMA Estimation," Journal of the American Statistical Association, 83, 490-498.

Hannan, E. J., And J. Rissanen (1982): "Recursive Estimation of Mixed AutoregressiveMoving Average Order," Biometrika, 69, 81-94.

Ibragimov, A. (1962): "Some Limit Theorems for Stationary Processes," Theory of Probability and its Applications, 7, 349-382.

Kapetanios, G. (2003): "A Note on an Iterative Least Squares Estimation Method for ARMA and VARMA models," Economics Letters, 79(3), 305-312.

Kascha, C. (2012): "A Comparison of Estimation Methods for Vector Autoregressive Moving-Average Models," Econometric Reviews, 31, 297-324.

Koreisha, S., and T. Pukkila (1990): "A Generalized Least Squares Approach for Estimation of Autoregressive Moving-Average Models," Journal of Time Series Analysis, $11,139-151$.

- (2004): "The Specification of Vector Autoregressive Moving Average Models," Journal of Statistical Computation and Simulation, 74, 547-565.

Lippi, M., And L. Reichlin (1994): "VAR Analysis, Nonfundamental Representations, Blaschke Matrices," Journal of Econometrics, 63(1), 307 - 325.

LÜtкepohl, H. (2007): New Introduction to Multiple Time Series Analysis. SpringerVerlag.

LÜtkepohl, H., And D. S. Poskitt (1996): "Specification of Echelon-Form VARMA Models," Journal of Business \& Economic Statistics, 14, 69-79.

NG, S., And P. Perron (1995): "Unit Root Tests in ARMA Models with Data-Dependent Methods for the Selection of the Truncation Lag," Journal of the American Statistical Association, 90, 268-281.

Pastorello, S., V. Patilea, and E. Renault (2003): "Iterative and Recursive Estimation in Structural Nonadaptive Models," Journal of Business and Economics Statistics, 21(460), 449-509. 
PoskitT, D. S. (1992): "Identification of Echelon Canonical Forms for Vector Linear Processes Using Least Squares," The Annals of Statistics, 20(1), 195-215.

Romano, J. P., And L. A. Thombs (1996): "Inference For Autocorrelations Under Weak Assumptions," Journal of the American Statistical Association, 91, 590-600.

Rubio-Ramírez, J. F., D. F. Waggoner, and T. Zha (2010): "Structural Vector Autoregressions: Theory of Identification and Algorithms for Inference," The Review of Economic Studies, 77, 665-696.

SpliId, H. (1983): "A Fast Estimation Method for the Vector Autoregressive Moving Average Model With Exogenous Variables," Journal of the American Statistical Association, $78,843-849$.

Stock, J. H., and M. W. Watson (2002a): "Forecasting Using Principal Components From a Large Number of Predictors," Journal of the American Statistical Association, 97(460), 1167-1179.

Stock, J. H., And M. W. Watson (2002b): "Macroeconomic Forecasting Using Diffusion Indexes," Journal of Business and Economics Statistics, 20, 147-162.

Stock, J. H., and M. W. Watson (2006): "Forecasting with Many Predictors," vol. 1 of Handbook of Economic Forecasting, chap. 10, pp. 515 - 554. Elsevier.

Tibshirani, R. (1996): "Regression Shrinkage and Selection via the Lasso," Journal of the Royal Statistical Society. Series B (Methodological), 58(1), pp. 267-288.

Uhlig, H. (2005): "What are the effects of monetary policy on output? Results from an agnostic identification procedure," Journal of Monetary Economics, 52(2), 381 - 419.

\section{Appendix}

Proof of Theorem 1: Denote $\phi=\left(\phi_{1}, \phi_{2}, \ldots, \phi_{n}\right)^{\prime}$ with $\phi \in \mathbb{B}$, as an n-dimensional vector collecting the parameter estimates of a general weak $\operatorname{VARMA}(\mathrm{p}, \mathrm{q})$ model. We start proving the consistency of the IOLS estimator. This proof follows analogous steps as in Theorems 2 and 4 in Dominitz and Sherman (2005) (DS henceforth). The steps in our proof are related to the primitive conditions in these theorems and hence we refer to them 
to easy the exposition. From DS, if $N(\phi)$ is an $\mathrm{ACM}$ on $\left(\mathbb{B}, E_{n}\right)$, then $N(\phi)$ is also a contraction mapping. Item i. in Assumption B.3 implies that $N\left(\beta^{j}\right)$ is an ACM, so that $\left|N\left(\beta^{j-1}\right)-N(\beta)\right| \leq \kappa\left|\beta^{j-1}-\beta\right|$ and $N(\beta)=\beta$ hold. From Lemma 5, the sample mapping is an $\mathrm{ACM}$ on $\left(\mathbb{B}, E_{n}\right)$ with a fixed point $\widehat{\beta}$ in the closed set $\mathbb{B}, \widehat{\beta} \in \mathbb{B}$ (condition ii. in DS's Theorem 4). First, bound $|\widehat{\beta}-\beta|$ as

$$
|\widehat{\beta}-\beta| \leq\left|\beta^{j}-\beta\right|+\left|\widehat{\beta}-\beta^{j}\right|
$$

To show that $\left|\beta^{j}-\beta\right|$ converges to zero, rewrite it as

$$
\left|\beta^{j}-\beta\right|=\left|N\left(\beta^{j-1}\right)-N(\beta)\right| \leq \kappa\left|\beta^{j-1}-\beta\right| .
$$

Recursive substitution of (21) yields $\left|\beta^{j}-\beta\right| \leq \kappa^{j}\left|\beta^{0}-\beta\right|$. As $j \longrightarrow \infty,\left|\beta^{j}-\beta\right|=o$ (1), and hence the first term on the right-hand side of (20) converges to zero. It remains to show that $\left|\widehat{\beta}-\beta^{j}\right|$ has order $o_{p}(1)$. We bound $\left|\widehat{\beta}-\beta^{j}\right|$ using the auxiliary result given by (S.46) in Lemma 5, so that

$$
\left|\widehat{\beta}-\beta^{j}\right| \leq \kappa^{j}\left|\beta^{0}-\widehat{\beta}\right|+\left(\sum_{i=0}^{j-1}\right) \kappa^{i}\left[\sup _{\phi \in \mathbb{B}}\left|\widehat{N}_{T}(\phi)-N(\phi)\right|\right],
$$

where $\beta^{0}$ is the vector collecting the initial estimates for the population mapping. As $j \longrightarrow \infty$, with $\kappa \in(0,1], \beta^{0} \in \mathbb{B}, \widehat{\beta} \in \mathbb{B}$, and $\mathbb{B}$ is a closed ball centered at $\beta$, it follows that (22) reduces to

$$
\left|\widehat{\beta}-\beta^{j}\right| \leq \sup _{\phi \in \mathbb{B}}\left|\widehat{N}_{T}(\phi)-N(\phi)\right|\left[\frac{1}{1-\kappa}\right]
$$

Because the second term in brackets on the right-hand side of (23) is bounded and the first term has order $o_{p}(1)$, (Lemma 3$),\left|\widehat{\beta}-\beta^{j}\right|=o_{p}(1)$, which implies $|\widehat{\beta}-\beta|=o_{p}(1)$ and completes the proof of the consistency result. The consistency result is equivalent to condition i. in DS's Theorem 4 , as $\widehat{\beta}^{j}$ converges uniformly in $\mathbb{B}$ to $\widehat{\beta}$ (Lemma 5).

We now turn our attention to the asymptotic distribution of the IOLS estimator. First, write

$$
\sqrt{T}\left|\widehat{\beta}^{j}-\beta\right| \leq \sqrt{T}|\widehat{\beta}-\beta|+\sqrt{T}\left|\widehat{\beta}^{j}-\widehat{\beta}\right|
$$


Lemma 6 gives that the second term on the right-hand side of $(24)$ is $o_{p}(1)$ as $j, T \longrightarrow \infty$ and $\frac{\ln (T)}{j}=o(1)$ (condition iii. in DS's Theorem 4). Next, rewrite $\sqrt{T}[\widehat{\beta}-\beta]$ as

$\sqrt{T}[\widehat{\beta}-\beta]=\sqrt{T}\left[\widehat{N}_{T}(\beta)-\breve{N}_{T}(\beta)\right]+\sqrt{T}\left[\widehat{N}_{T}(\widehat{\beta})-\widehat{N}_{T}(\beta)\right]+\sqrt{T}\left[\breve{N}_{T}(\beta)-\beta\right]$.

Lemma 2 gives $\sqrt{T}\left[\widehat{N}_{T}(\beta)-\breve{N}_{T}(\beta)\right]=O_{p}\left(T^{-1 / 2}\right)$. Using the mean value theorem, rewrite the second term on the right-hand side of $(25)$ as

$$
\begin{array}{r}
\sqrt{T}\left[\widehat{N}_{T}(\widehat{\beta})-\widehat{N}_{T}(\beta)\right]=\sqrt{T}\left\{\left[\widehat{N}_{T}(\widehat{\beta})-\breve{N}_{T}(\widehat{\beta})\right]+\left[\breve{N}_{T}(\beta)-\widehat{N}_{T}(\beta)\right]\right\}+ \\
\sqrt{T}\left\{\breve{\Lambda}_{T}(\widehat{\beta}, \beta)[\widehat{\beta}-\beta]\right\},
\end{array}
$$

where $\breve{\Lambda}_{T}(\widehat{\beta}, \beta)=\int_{0}^{1} \breve{V}_{T}(\widehat{\beta}+\xi(\widehat{\beta}-\beta)) d \xi$. Because the first term has order $O_{p}\left(T^{-1 / 2}\right)$ and $\breve{\Lambda}_{T}(\widehat{\beta}, \beta)[\widehat{\beta}-\beta]$ converges uniformly to its population counterpart (Lemma 4), (25) reads

$\sqrt{T}[\widehat{\beta}-\beta]=\sqrt{T}\left[\left[I_{n}-\Lambda(\widehat{\beta}, \beta)\right]^{-1}\left[\breve{N}_{T}(\beta)-\beta\right]\right]$.

Notably, Lemma 4 fulfills the condition v. in DS's Theorem 4. From item (i.) in Theorem 1, $\widehat{\beta}$ converges in probability to $\beta$ as $j \longrightarrow \infty$ with $T \longrightarrow \infty$, implying that $\Lambda(\widehat{\beta}, \beta)$ converges in probability to $V(\beta)$. It follows that $(27)$ reduces to

$\sqrt{T}[\widehat{\beta}-\beta]=\sqrt{T}\left[\left[I_{n}-V(\beta)\right]^{-1}\left[\breve{N}_{T}(\beta)-\beta\right]\right]$.

The next step consists of proving condition iv. in DS's Theorem 4 . As $\breve{N}_{T}(\beta)$ is evaluated at the true vector of parameters and $T \longrightarrow \infty$, it follows that $\sqrt{T}\left[\breve{N}_{T}(\beta)-\beta\right]$ reads

$$
\begin{aligned}
\sqrt{T}\left[\breve{N}_{T}(\beta)-\beta\right]=\left[R^{\prime}\left[\left(\frac{1}{T} \sum_{t=\bar{q}+1}^{T} X_{t} X_{t}^{\prime}\right) \otimes I_{K}\right] R\right]^{-1} \times & {\left[\frac{1}{\sqrt{T}} \sum_{t=\bar{q}+1}^{T} R^{\prime}\left(X_{t} \otimes I_{K}\right) U_{t}\right] . }
\end{aligned}
$$

Recall that the last term is not an m.d.s, because $U_{t}$ is not an i.i.d. process. Assumption B.1 allows a $\operatorname{VMA}(\infty)$ representation of $Y_{t}$ of the form $Y_{t}=\sum_{i=0}^{\infty} \Theta_{i} U_{t-i}$ with $\Theta_{0}=I_{K}$. As discussed in Francq and Zakoian (1998), a stationary process which is a function of a finite 
number of current and lagged values of $U_{t}$ satisfies a mixing property of the form Assumption B.2. Using the $\operatorname{VMA}(\infty)$ representation of $Y_{t}$, partition $X_{t}$ in (29) into $X_{t}=X_{t}^{r}+X_{t}^{r+}$, such that

$$
X_{t}=\left(\begin{array}{c}
Y_{t}-U_{t} \\
Y_{t-1} \\
\vdots \\
Y_{t-p} \\
U_{t-1} \\
\vdots \\
U_{t-q}
\end{array}\right)=\left(\begin{array}{c}
\sum_{i=1}^{r} \Theta_{i} U_{t-i} \\
\sum_{i=0}^{r} \Theta_{i} U_{t-1-i} \\
\vdots \\
\sum_{i=0}^{r} \Theta_{i} U_{t-p-i} \\
U_{t-1} \\
\vdots \\
U_{t-q}
\end{array}\right)+\left(\begin{array}{c}
\sum_{i=r+1}^{\infty} \Theta_{i} U_{t-i} \\
\sum_{i=r+1}^{\infty} \Theta_{i} U_{t-1-i} \\
\vdots \\
\sum_{i=r+1}^{\infty} \Theta_{i} U_{t-p-i} \\
0 \\
\vdots \\
0
\end{array}\right)=X_{t}^{r}+X_{t}^{r+}
$$

Rewrite $\frac{1}{\sqrt{T}} \sum_{t=\bar{q}+1}^{T} R^{\prime}\left(X_{t} \otimes I_{K}\right) U_{t}$ as

$\frac{1}{\sqrt{T}} \sum_{t=\bar{q}+1}^{T} R^{\prime}\left(X_{t} \otimes I_{K}\right) U_{t}=\frac{1}{\sqrt{T}} \sum_{t=\bar{q}+1}^{T} R^{\prime}\left(X_{t}^{r} \otimes I_{K}\right) U_{t}+\frac{1}{\sqrt{T}} \sum_{t=\bar{q}+1}^{T} R^{\prime}\left(X_{t}^{r+} \otimes I_{K}\right) U_{t}$.

Auxiliary results in Dufour and Pelletier (2014, Theorem 4.2) show that the second term on the right-hand side of (31) converges uniformly to zero in $T$ as $r \rightarrow \infty$. It follows that first term on the right-hand side of (31) satisfies the strong mixing conditions of the form Assumption B.2. We are now in position to use Ibragimov's 1962 central limit theorem for strong mixing processes (see also Dufour and Pelletier (2014, Lemma A.2)). This yields $\frac{1}{\sqrt{T}} \sum_{t=1}^{T} R^{\prime}\left(X_{t}^{r} \otimes I_{K}\right) U_{t} \stackrel{d}{\longrightarrow} \mathcal{N}\left(0, \mathcal{I}_{r}\right)$. From Francq and Zakoian, 1998, p. 157, $\mathcal{I}_{r} \stackrel{p}{\longrightarrow} \mathcal{I}$ as $r \rightarrow \infty$, such that as $T, r \rightarrow \infty$

$\sqrt{T}\left[\breve{N}_{T}(\beta)-\beta\right] \stackrel{d}{\longrightarrow} \mathcal{N}\left(0, Z \mathcal{I} Z^{\prime}\right)$

where $\mathcal{I}=\sum_{\ell=-\infty}^{\infty} \mathbb{E}\left\{\left[R^{\prime}\left(X_{t} \otimes I_{K}\right) U_{t}\right]\left[R^{\prime}\left(X_{t-\ell} \otimes I_{K}\right) U_{t-\ell}\right]^{\prime}\right\}$, and $Z=\left[R^{\prime}\left(H \otimes I_{K}\right) R\right]^{-1}$ with $H=\operatorname{plim} \frac{1}{T} \sum_{t=\bar{q}+1}^{T} X_{t} X_{t}^{\prime}$. The final result of this Theorem is obtained by combining the first element of the right-hand side of (28) with (32), such that

$\sqrt{T}[\widehat{\beta}-\beta] \stackrel{d}{\longrightarrow} \mathcal{N}\left(0, J Z \mathcal{I} Z^{\prime} J^{\prime}\right)$ 
where $J=\left[I_{n}-V(\beta)\right]^{-1}$.

Proof of Corollary 1: The consistency result follows directly from item i. in Theorem 1. To show item ii., rewrite $\sqrt{T}[\widehat{\beta}-\beta]$ using the same arguments as in the proof of Theorem 1 , such that

$\sqrt{T}[\widehat{\beta}-\beta]=\sqrt{T}\left[\left[I_{n}-V(\beta)\right]^{-1}\left[\breve{N}_{T}(\beta)-\beta\right]\right]$.

The limiting distribution of (34) depends on the limiting behaviour of,

$\sqrt{T}\left[\breve{N}_{T}(\beta)-\beta\right]=\left[R^{\prime}\left[\left(\frac{1}{T} \sum_{t=\bar{q}+1}^{T} X_{t} X_{t}^{\prime}\right) \otimes I_{K}\right] R\right]^{-1}\left[\frac{1}{\sqrt{T}} \sum_{t=\bar{q}+1}^{T} R^{\prime}\left(X_{t} \otimes I_{K}\right) U_{t}\right]$.

Because $U_{t}$ is an i.i.d. process, it follows that $R^{\prime}\left(X_{t} \otimes I_{K}\right) U_{t}$ is an m.d.s, in that the central limit theorem for m.d.s can be used to show that (35) converges in distribution to

$\sqrt{T}\left[\breve{N}_{T}(\beta)-\beta\right] \stackrel{d}{\longrightarrow} \mathcal{N}\left(0, Z R^{\prime}\left(H \otimes \Sigma_{u}\right) R Z^{\prime}\right)$

where $Z=\left[R^{\prime}\left(H \otimes I_{K}\right) R\right]^{-1}$ and $H=\operatorname{plim} \frac{1}{T} \sum_{t=\bar{q}+1}^{T} X_{t} X_{t}^{\prime}$. Define $J=\left[I_{n}-V(\beta)\right]^{-1}$ and combine (34) with (36), and the asymptotic distribution of the IOLS estimator for the strong $\operatorname{VARMA}(\mathrm{p}, \mathrm{q})$ models reads,

$\sqrt{T}[\widehat{\beta}-\beta] \stackrel{d}{\longrightarrow} \mathcal{N}\left(0, J Z R^{\prime}\left(H \otimes \Sigma_{u}\right) R Z^{\prime} J^{\prime}\right)$.

Lemma 1 Assume Assumptions B.1 and B.2 hold, then $\widehat{V}_{T}\left(\widehat{\beta}^{j}\right)=\frac{\partial \widehat{N}_{T}\left(\widehat{\beta}^{j}\right)}{\partial \widehat{\beta}^{j \prime}}$ is given by:

$$
\begin{aligned}
\widehat{V}_{T}\left(\widehat{\beta}^{j}\right)=\left\{\left[I_{1} \otimes W^{j^{-1}}\right]\right. & \frac{1}{T-\bar{q}} \sum_{t=1+\bar{q}}^{T}\left\{\left(Y_{t}^{\prime} \otimes I_{n}\right)\left(I_{K} \otimes R^{\prime}\right) \times\right. \\
{\left.\left[\left(I_{1} \otimes \mathbb{K}_{K, f} \otimes I_{K}\right)\left(I_{f} \otimes \operatorname{vec}\left(I_{K}\right)\right)\right] \frac{\partial \operatorname{vec}\left(\widehat{X}_{t}^{j}\right)}{\partial \widehat{\beta}^{j \prime}}\right\}+ } & \left\{\left[\left(\frac{1}{T-\bar{q}} \sum_{t=1+\bar{q}}^{T} \tilde{X}_{t}^{j \prime} Y_{t}\right)^{\prime} \otimes I_{n}\right]\left[-\left(W^{j}\right)^{-1} \otimes\left(W^{j}\right)^{-1}\right] \times\right. \\
\{ & {\left[\frac { 1 } { T - \overline { q } } \sum _ { t = 1 + \overline { q } } ^ { T } \left\{\left[\left(I_{n^{2}}+\mathbb{K}_{n, n}\right)\left(I_{n} \otimes \tilde{X}_{t}^{j \prime}\right)\left(R^{\prime} \otimes I_{K}\right) \times\right.\right.\right.} \\
& {\left.\left.\left.\left[\left(I_{f} \otimes \mathbb{K}_{K, 1} \otimes I_{K}\right)\left(I_{f} \otimes \operatorname{vec}\left(I_{K}\right)\right)\right] \frac{\partial \operatorname{vec}\left(\widehat{X}_{t}^{j \prime}\right)}{\partial \widehat{\beta}^{j \prime}}\right\}\right]\right\} }
\end{aligned}
$$


where $\mathbb{K}$ is the commutation matrix, $f=K(p+q+1), \quad \bar{q}=\max \{p, q\}$, $W^{j}=\left(\frac{1}{T-\bar{q}} \sum_{t=1+\bar{q}}^{T} \tilde{X}_{t}^{j \prime} \tilde{X}_{t}^{j}\right)$, and $\frac{\partial \operatorname{vec}\left(\widehat{X}_{t}^{j}\right)}{\partial \widehat{\beta}^{j \prime}}=\frac{\partial \operatorname{vec}\left(\widehat{X}_{t}^{j \prime}\right)}{\partial \widehat{\beta}^{j \prime}}$ with

$$
\begin{gathered}
\frac{\partial \operatorname{vec}\left(\widehat{X}_{t}^{j}\right)}{\partial \widehat{\beta}^{j^{\prime}}}=\operatorname{vec}\left(-\frac{\partial \widehat{U}_{t}^{j}}{\partial \widehat{\beta}^{j^{\prime}}}, 0_{K, n}, \cdots 0_{K, n}, \frac{\partial \widehat{U}_{t-1}^{j}}{\partial \widehat{\beta}^{j^{\prime}}}, \cdots, \frac{\partial \widehat{U}_{t-q}^{j}}{\partial \widehat{\beta}^{j^{\prime}}}\right) \quad \text { and } \\
\frac{\partial \widehat{U}_{t}^{j}}{\partial \widehat{\beta}^{j^{\prime}}}=\left\{\left(\widehat{A}_{0}^{j^{-1}}\left[\widehat{A}_{1}^{j} Y_{t-1}+\ldots+\widehat{A}_{p}^{j} Y_{t-p}+\widehat{M}_{1}^{j} \widehat{U}_{t-1}^{j}+\ldots+\widehat{M}_{q}^{j} \widehat{U}_{t-q}^{j}\right]\right)^{\prime} \otimes \widehat{A}_{0}^{j^{-1}}\right\} \times \\
{\left[I_{K^{2}}: 0: \ldots: 0\right] R-\left[\left(Y_{t-1}^{\prime}, \ldots, Y_{t-p}^{\prime}, \widehat{U}_{t-1}^{j \prime}, \ldots, \widehat{U}_{t-q}^{j \prime}\right) \otimes \widehat{A}_{0}^{j^{-1}}\right]\left[0: I_{K^{2}(p+q)}\right] R-} \\
\widehat{A}_{0}^{j-1}\left[M_{1}^{j} \frac{\partial \widehat{U}_{t-1}^{j}}{\partial \widehat{\beta}^{j^{\prime}}}+\ldots+M_{q}^{j} \frac{\partial \widehat{U}_{t-q}^{j}}{\partial \widehat{\beta}^{j^{\prime}}}\right] .
\end{gathered}
$$

Proof. See Section S.1 in the online Supplement.

Lemma 2 Assume Assumptions B.1 and B.2 hold. Then,

$\sup _{\phi \in \mathbb{B}}\left\|\widehat{N}_{T}(\phi)-\breve{N}_{T}(\phi)\right\|=O_{p}\left(T^{-1}\right)$.

Proof. See Section S.1 in the online Supplement.

Lemma 3 Assume Assumptions B.1, B.2, and B.3 hold. Then,

$\sup _{\phi \in \mathbb{B}}\left\|\widehat{N}_{T}(\phi)-N(\phi)\right\|=o_{p}(1)$ as $T \longrightarrow \infty$.

Proof. See Section S.1 in the online Supplement.

Lemma 4 Assume Assumptions B.1, B.2, and B.3 hold. Then,

$\sup _{\phi, \gamma \in \mathbb{B}}\left\|\left[\widehat{\Lambda}_{T}(\phi, \gamma)-\Lambda(\phi, \gamma)\right](\phi-\gamma)\right\|=o_{p}(1)$ as $T \longrightarrow \infty$,

$\sup _{\phi, \gamma \in \mathbb{B}}\left\|\left[\breve{\Lambda}_{T}(\phi, \gamma)-\Lambda(\phi, \gamma)\right](\phi-\gamma)\right\|=o_{p}(1)$ as $T \longrightarrow \infty$.

Proof. See Section S.1 in the online Supplement.

Lemma 5 Assume Assumptions B.1, B.2, and B.3 hold, then, $\widehat{N}_{T}(\phi)$ is an ACM on $\left(\mathbb{B}, E_{n}\right)$, with $\phi \in \mathbb{B}$ and it has fixed point denoted by $\widehat{\beta}$, such that $\left|\widehat{\beta}^{j}-\widehat{\beta}\right|=o_{p}(1)$ uniformly in $\mathbb{B}$ as $j, T \longrightarrow \infty$.

Proof. See Section S.1 in the online Supplement.

Lemma 6 Assume Assumptions B.1, B.2, and B.3 hold. If

i. $\widehat{N}_{T}(\phi)$ is an $A C M$ on $\left(\mathbb{B}, E_{n}\right)$

then, $\sqrt{T}\left|\widehat{\beta}^{j}-\widehat{\beta}\right|=o_{p}(1)$ as $j, T \longrightarrow \infty$ and $\frac{\ln (T)}{j}=o(1)$.

Proof. See Section S.1 in the online Supplement. 


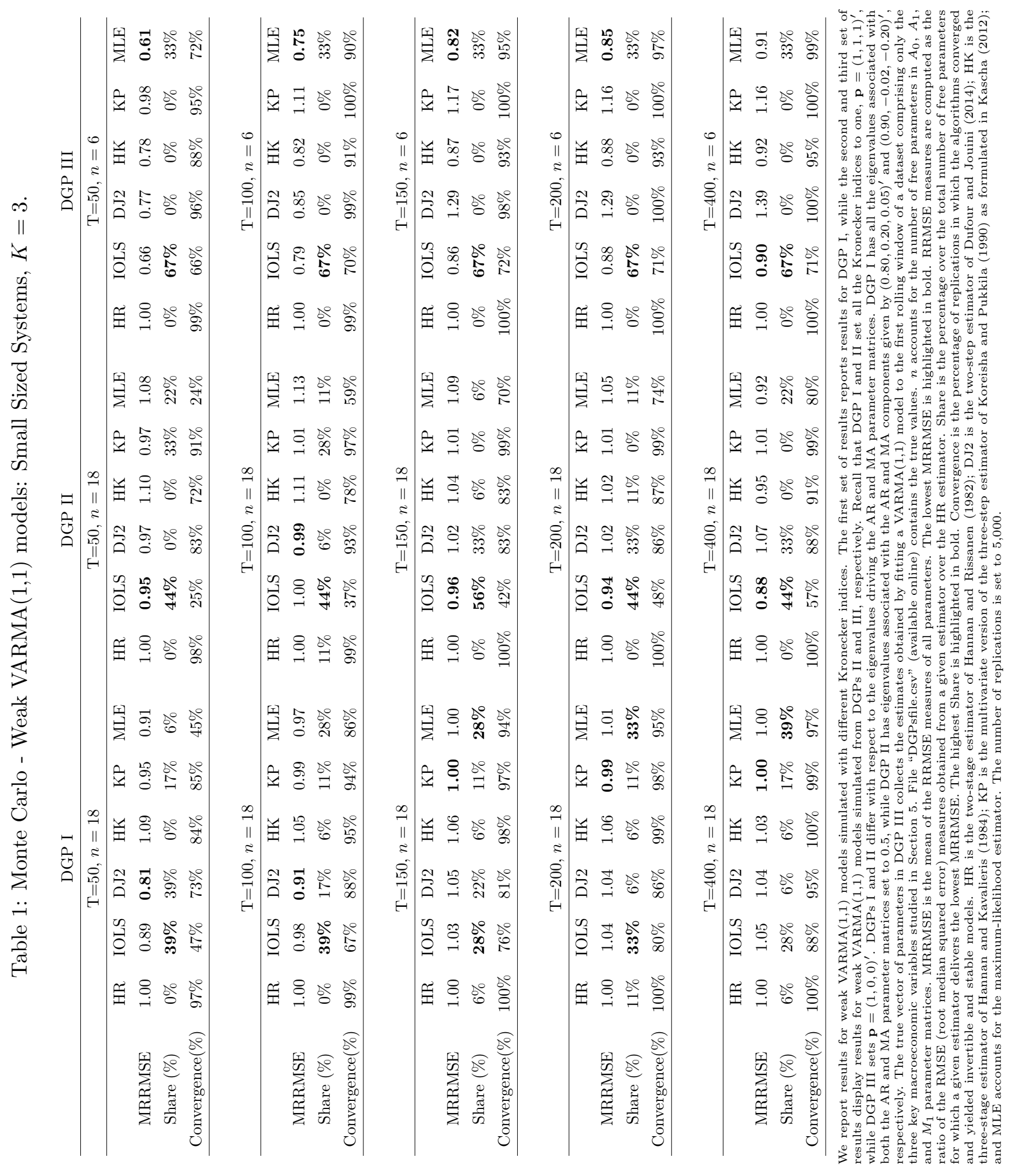




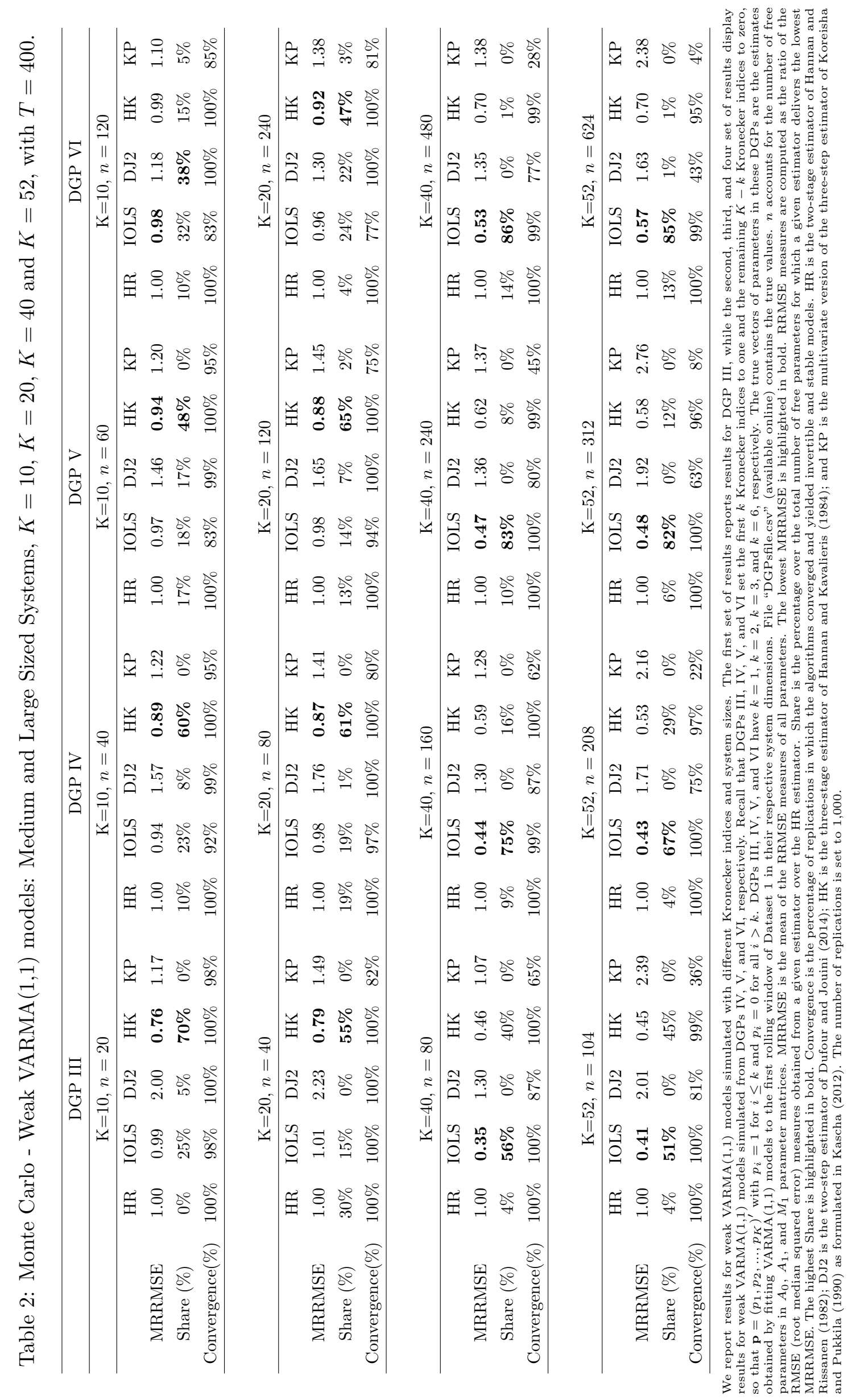


Table 3: Forecast Summary: VARMA Out-of-Sample Performance Relative to Alternative Group of Models

\begin{tabular}{cccccccccccccccc}
\multicolumn{10}{c}{$K=3$} \\
\hline \multicolumn{10}{c}{ VARMA } \\
\hline AR & ARMA & VAR & FM & BVAR & AR & ARMA & VAR & FM & BVAR & AR & ARMA & VAR & FM & BVAR \\
Hor: 1 & $\mathbf{1 0 0 \%}$ & $\mathbf{1 0 0 \%}$ & $\mathbf{1 0 0 \%}$ & $\mathbf{1 0 0 \%}$ & $\mathbf{1 0 0 \%}$ & $\mathbf{1 0 0 \%}$ & $\mathbf{1 0 0 \%}$ & $\mathbf{1 0 0 \%}$ & $\mathbf{1 0 0 \%}$ & $\mathbf{1 0 0 \%}$ & $\mathbf{6 7 \%}$ & $\mathbf{1 0 0 \%}$ & $\mathbf{1 0 0 \%}$ & $\mathbf{1 0 0 \%}$ & $33 \%$ \\
Hor: 2 & $\mathbf{1 0 0 \%}$ & $\mathbf{1 0 0 \%}$ & $\mathbf{1 0 0 \%}$ & $\mathbf{1 0 0 \%}$ & $\mathbf{1 0 0 \%}$ & $\mathbf{1 0 0 \%}$ & $\mathbf{6 7 \%}$ & $\mathbf{1 0 0 \%}$ & $\mathbf{1 0 0 \%}$ & $\mathbf{1 0 0 \%}$ & $\mathbf{6 7 \%}$ & $\mathbf{6 7 \%}$ & $\mathbf{1 0 0 \%}$ & $\mathbf{1 0 0 \%}$ & $33 \%$ \\
Hor: 3 & $\mathbf{1 0 0 \%}$ & $\mathbf{6 7 \%}$ & $\mathbf{1 0 0 \%}$ & $\mathbf{1 0 0 \%}$ & $\mathbf{1 0 0 \%}$ & $\mathbf{6 7 \%}$ & $33 \%$ & $\mathbf{6 7 \%}$ & $\mathbf{1 0 0 \%}$ & $\mathbf{6 7 \%}$ & $\mathbf{1 0 0 \%}$ & $33 \%$ & $\mathbf{1 0 0 \%}$ & $\mathbf{1 0 0 \%}$ & $\mathbf{6 7 \%}$ \\
Hor: 6 & $\mathbf{1 0 0 \%}$ & $\mathbf{1 0 0 \%}$ & $\mathbf{1 0 0 \%}$ & $\mathbf{1 0 0 \%}$ & $\mathbf{1 0 0 \%}$ & $\mathbf{1 0 0 \%}$ & $\mathbf{1 0 0 \%}$ & $\mathbf{6 7 \%}$ & $\mathbf{1 0 0 \%}$ & $\mathbf{1 0 0 \%}$ & $\mathbf{6 7 \%}$ & $33 \%$ & $33 \%$ & $\mathbf{6 7 \%}$ & $\mathbf{6 7 \%}$ \\
Hor: 9 & $\mathbf{6 7 \%}$ & $\mathbf{1 0 0 \%}$ & $\mathbf{1 0 0 \%}$ & $0 \%$ & $\mathbf{6 7 \%}$ & $\mathbf{6 7 \%}$ & $\mathbf{6 7 \%}$ & $\mathbf{1 0 0 \%}$ & $0 \%$ & $\mathbf{6 7 \%}$ & $0 \%$ & $0 \%$ & $0 \%$ & $0 \%$ & $0 \%$ \\
Hor: 12 & $\mathbf{6 7 \%}$ & $\mathbf{6 7 \%}$ & $\mathbf{6 7 \%}$ & $33 \%$ & $\mathbf{6 7 \%}$ & $\mathbf{6 7 \%}$ & $33 \%$ & $33 \%$ & $33 \%$ & $33 \%$ & $33 \%$ & $33 \%$ & $0 \%$ & $0 \%$ & $33 \%$ \\
\hline
\end{tabular}

\begin{tabular}{cccccccccccccccc}
\multicolumn{10}{c}{$K=10$} \\
\hline \multicolumn{10}{c}{ VARMA } \\
\hline AR & ARMA & VAR & FM & BVAR & AR & ARMA & VAR & FM & BVAR & AR & ARMA & VAR & FM & BVAR \\
Hor: 1 & $\mathbf{8 3 \%}$ & $\mathbf{9 2 \%}$ & $\mathbf{1 0 0 \%}$ & $\mathbf{6 7 \%}$ & $42 \%$ & $\mathbf{8 3 \%}$ & $\mathbf{8 3 \%}$ & $\mathbf{1 0 0 \%}$ & $\mathbf{5 8 \%}$ & $42 \%$ & $\mathbf{5 0 \%}$ & $\mathbf{5 8 \%}$ & $\mathbf{9 2 \%}$ & $25 \%$ & $17 \%$ \\
Hor: 2 & $\mathbf{1 0 0 \%}$ & $\mathbf{8 3 \%}$ & $\mathbf{1 0 0 \%}$ & $\mathbf{9 2 \%}$ & $\mathbf{1 0 0 \%}$ & $\mathbf{9 2 \%}$ & $\mathbf{7 5 \%}$ & $\mathbf{1 0 0 \%}$ & $\mathbf{8 3 \%}$ & $\mathbf{9 2 \%}$ & $\mathbf{6 7 \%}$ & $\mathbf{5 8 \%}$ & $\mathbf{9 2 \%}$ & $\mathbf{6 7 \%}$ & $42 \%$ \\
Hor: 3 & $\mathbf{1 0 0 \%}$ & $\mathbf{5 0 \%}$ & $\mathbf{1 0 0 \%}$ & $\mathbf{9 2 \%}$ & $\mathbf{1 0 0 \%}$ & $\mathbf{8 3 \%}$ & $33 \%$ & $\mathbf{8 3 \%}$ & $\mathbf{7 5 \%}$ & $\mathbf{5 8 \%}$ & $\mathbf{5 0 \%}$ & $25 \%$ & $\mathbf{7 5 \%}$ & $42 \%$ & $25 \%$ \\
Hor: 6 & $\mathbf{9 2 \%}$ & $\mathbf{1 0 0 \%}$ & $\mathbf{9 2 \%}$ & $\mathbf{1 0 0 \%}$ & $\mathbf{1 0 0 \%}$ & $\mathbf{1 0 0 \%}$ & $\mathbf{8 3 \%}$ & $\mathbf{6 7 \%}$ & $\mathbf{1 0 0 \%}$ & $\mathbf{5 0 \%}$ & $\mathbf{5 8 \%}$ & $\mathbf{5 8 \%}$ & $\mathbf{5 0 \%}$ & $\mathbf{7 5 \%}$ & $33 \%$ \\
Hor: 9 & $\mathbf{1 0 0 \%}$ & $\mathbf{9 2 \%}$ & $\mathbf{5 8 \%}$ & $33 \%$ & $\mathbf{8 3 \%}$ & $\mathbf{1 0 0 \%}$ & $\mathbf{9 2 \%}$ & $\mathbf{5 0 \%}$ & $17 \%$ & $\mathbf{5 0 \%}$ & $\mathbf{5 0 \%}$ & $\mathbf{5 8 \%}$ & $8 \%$ & $25 \%$ & $33 \%$ \\
Hor: 12 & $\mathbf{1 0 0 \%}$ & $\mathbf{9 2 \%}$ & $\mathbf{6 7 \%}$ & $\mathbf{6 7 \%}$ & $\mathbf{9 2 \%}$ & $\mathbf{5 8 \%}$ & $\mathbf{5 8 \%}$ & $\mathbf{5 0 \%}$ & $\mathbf{5 8 \%}$ & $\mathbf{7 5 \%}$ & $33 \%$ & $33 \%$ & $33 \%$ & $17 \%$ & $42 \%$ \\
\hline
\end{tabular}

\begin{tabular}{|c|c|c|c|c|c|c|c|c|c|c|c|c|c|c|c|}
\hline \multicolumn{16}{|c|}{$K=20$} \\
\hline & \multicolumn{5}{|c|}{ VARMA } & \multicolumn{5}{|c|}{$\mathbf{p}_{k=1}$} & \multicolumn{5}{|c|}{$\mathbf{p}_{L P}$} \\
\hline & $\mathrm{AR}$ & ARMA & VAR & FM & BVAR & $\mathrm{AR}$ & ARMA & VAR & FM & BVAR & $\mathrm{AR}$ & ARMA & VAR & FM & BVAR \\
\hline Hor: 1 & $75 \%$ & $67 \%$ & $100 \%$ & $75 \%$ & $75 \%$ & $67 \%$ & $58 \%$ & $92 \%$ & $58 \%$ & $67 \%$ & $58 \%$ & $33 \%$ & $75 \%$ & $50 \%$ & $33 \%$ \\
\hline Hor: 2 & $100 \%$ & $75 \%$ & $100 \%$ & $75 \%$ & $100 \%$ & $75 \%$ & $58 \%$ & $92 \%$ & $67 \%$ & $83 \%$ & $75 \%$ & $50 \%$ & $83 \%$ & $50 \%$ & $75 \%$ \\
\hline Hor: 3 & $100 \%$ & $42 \%$ & $100 \%$ & $67 \%$ & $92 \%$ & $83 \%$ & $33 \%$ & $58 \%$ & $17 \%$ & $50 \%$ & $67 \%$ & $33 \%$ & $58 \%$ & $42 \%$ & $58 \%$ \\
\hline Hor: 6 & $83 \%$ & $100 \%$ & $75 \%$ & $100 \%$ & $83 \%$ & $100 \%$ & $75 \%$ & $58 \%$ & $100 \%$ & $50 \%$ & $83 \%$ & $67 \%$ & $58 \%$ & $67 \%$ & $50 \%$ \\
\hline Hor: 9 & $100 \%$ & $83 \%$ & $75 \%$ & $33 \%$ & $83 \%$ & $100 \%$ & $75 \%$ & $67 \%$ & $33 \%$ & $58 \%$ & $83 \%$ & $75 \%$ & $58 \%$ & $25 \%$ & $67 \%$ \\
\hline Hor: 12 & $100 \%$ & $75 \%$ & $75 \%$ & $42 \%$ & $100 \%$ & $58 \%$ & $67 \%$ & $58 \%$ & $42 \%$ & $42 \%$ & $42 \%$ & $42 \%$ & $33 \%$ & $42 \%$ & $25 \%$ \\
\hline
\end{tabular}

\begin{tabular}{ccccccccccccccccc}
\multicolumn{10}{c}{$K=40$} \\
\hline \multicolumn{10}{c}{ VARMA } \\
\hline & AR & ARMA & VAR & FM & BVAR & AR & ARMA & VAR & FM & BVAR & AR & ARMA & VAR & FM & BVAR \\
Hor: 1 & $\mathbf{8 3 \%}$ & $\mathbf{7 5 \%}$ & $\mathbf{1 0 0 \%}$ & $\mathbf{6 7 \%}$ & $\mathbf{9 2 \%}$ & $\mathbf{6 7 \%}$ & $42 \%$ & $\mathbf{1 0 0 \%}$ & $33 \%$ & $\mathbf{6 7 \%}$ & $\mathbf{6 7 \%}$ & $\mathbf{6 7 \%}$ & $\mathbf{1 0 0 \%}$ & $\mathbf{5 0 \%}$ & $\mathbf{7 5 \%}$ \\
Hor: 2 & $\mathbf{8 3 \%}$ & $\mathbf{8 3 \%}$ & $\mathbf{1 0 0 \%}$ & $\mathbf{5 8 \%}$ & $\mathbf{9 2 \%}$ & $\mathbf{6 7 \%}$ & $\mathbf{5 0 \%}$ & $\mathbf{1 0 0 \%}$ & $33 \%$ & $\mathbf{7 5 \%}$ & $\mathbf{7 5 \%}$ & $\mathbf{6 7 \%}$ & $\mathbf{1 0 0 \%}$ & $\mathbf{5 0 \%}$ & $\mathbf{7 5 \%}$ \\
Hor: 3 & $\mathbf{1 0 0 \%}$ & $\mathbf{5 8 \%}$ & $\mathbf{1 0 0 \%}$ & $\mathbf{7 5 \%}$ & $\mathbf{9 2 \%}$ & $42 \%$ & $33 \%$ & $\mathbf{1 0 0 \%}$ & $17 \%$ & $42 \%$ & $\mathbf{6 7 \%}$ & $25 \%$ & $\mathbf{1 0 0 \%}$ & $\mathbf{5 8 \%}$ & $\mathbf{5 8 \%}$ \\
Hor: 6 & $\mathbf{9 2 \%}$ & $\mathbf{1 0 0 \%}$ & $\mathbf{7 5 \%}$ & $\mathbf{5 6 \%}$ & $\mathbf{6 7 \%}$ & $\mathbf{1 0 0 \%}$ & $\mathbf{6 7 \%}$ & $\mathbf{1 0 0 \%}$ & $42 \%$ & $42 \%$ & $\mathbf{6 7 \%}$ & $\mathbf{5 8 \%}$ & $\mathbf{1 0 0 \%}$ & $42 \%$ & $\mathbf{5 8 \%}$ \\
Hor: 9 & $\mathbf{1 0 0 \%}$ & $\mathbf{8 3 \%}$ & $\mathbf{1 0 0 \%}$ & $33 \%$ & $\mathbf{7 5 \%}$ & $\mathbf{1 0 0 \%}$ & $\mathbf{6 7 \%}$ & $\mathbf{1 0 0 \%}$ & $8 \%$ & $33 \%$ & $\mathbf{5 8 \%}$ & $42 \%$ & $\mathbf{1 0 0 \%}$ & $25 \%$ & $42 \%$ \\
Hor: 12 & $\mathbf{9 2 \%}$ & $\mathbf{8 3 \%}$ & $\mathbf{1 0 0 \%}$ & $\mathbf{5 8 \%}$ & $\mathbf{7 5 \%}$ & $33 \%$ & $\mathbf{6 7 \%}$ & $\mathbf{1 0 0 \%}$ & $42 \%$ & $42 \%$ & $42 \%$ & $\mathbf{5 8 \%}$ & $\mathbf{1 0 0 \%}$ & $\mathbf{5 8 \%}$ & $\mathbf{5 8 \%}$ \\
\hline
\end{tabular}

\begin{tabular}{|c|c|c|c|c|c|c|c|c|c|c|c|c|c|c|c|}
\hline \multicolumn{16}{|c|}{$K=52$} \\
\hline & \multicolumn{5}{|c|}{ VARMA } & \multicolumn{5}{|c|}{$\mathbf{p}_{k=1}$} & \multicolumn{5}{|c|}{$\mathbf{p}_{L P}$} \\
\hline & $\mathrm{AR}$ & ARMA & VAR & FM & BVAR & $\mathrm{AR}$ & ARMA & VAR & FM & BVAR & $\mathrm{AR}$ & ARMA & VAR & FM & BVAR \\
\hline Hor: 1 & $67 \%$ & $33 \%$ & $100 \%$ & $33 \%$ & $67 \%$ & $67 \%$ & $33 \%$ & $100 \%$ & $33 \%$ & $67 \%$ & $0 \%$ & $0 \%$ & $100 \%$ & $0 \%$ & $67 \%$ \\
\hline Hor: 2 & $67 \%$ & $33 \%$ & $100 \%$ & $33 \%$ & $67 \%$ & $67 \%$ & $33 \%$ & $100 \%$ & $33 \%$ & $67 \%$ & $33 \%$ & $33 \%$ & $100 \%$ & $33 \%$ & $33 \%$ \\
\hline Hor: 3 & $100 \%$ & $67 \%$ & $100 \%$ & $33 \%$ & $100 \%$ & $67 \%$ & $33 \%$ & $100 \%$ & $0 \%$ & $67 \%$ & $0 \%$ & $0 \%$ & $100 \%$ & $0 \%$ & $0 \%$ \\
\hline Hor: 6 & $100 \%$ & $67 \%$ & $100 \%$ & $67 \%$ & $67 \%$ & $100 \%$ & $67 \%$ & $100 \%$ & $67 \%$ & $33 \%$ & $0 \%$ & $0 \%$ & $100 \%$ & $0 \%$ & $0 \%$ \\
\hline Hor: 9 & $100 \%$ & $67 \%$ & $100 \%$ & $33 \%$ & $67 \%$ & $100 \%$ & $67 \%$ & $100 \%$ & $0 \%$ & $33 \%$ & $0 \%$ & $0 \%$ & $100 \%$ & $0 \%$ & $0 \%$ \\
\hline Hor: 12 & $67 \%$ & $100 \%$ & $100 \%$ & $67 \%$ & $67 \%$ & $33 \%$ & $67 \%$ & $100 \%$ & $33 \%$ & $33 \%$ & $33 \%$ & $33 \%$ & $100 \%$ & $67 \%$ & $33 \%$ \\
\hline
\end{tabular}

Hor:1, Hor: 2, Hor: 3, Hor: 6, Hor: 9, and Hor: 12 account for one-, two- three- six-, nine-, and twelve-month-ahead forecast, respectively. For each system size, the first panel reports the frequency (in percentage points) for which at least one of the VARMA specifications ( $\mathbf{p}_{k=1}, \mathbf{p}_{k}=2, \mathbf{p}_{k}=3$, $\mathbf{p}_{k=4}, \mathbf{p}_{k=5}, \mathbf{p}_{k=6}, \mathbf{p}_{L P}$, and $\mathbf{p}_{H K}$ ) outperforms (delivers the lowest RelMSFE measures) the assigned group of competitors in a given forecast horizon. The second and third panel report the frequencies for which the $\mathbf{p}_{k=1}$ and $\mathbf{p}_{L P}$ specifications, respectively, outperform the assigned group of competitors. We consider five groups of competitors. AR collects the AR(1) model; ARMA has the ARMA(1,1) specifications estimated with the IOLS and MLE estimators, VAR contains the VAR $\left(p^{*}\right)$ model, where $p^{*}$ is obtained by minimizing the AIC criterion; FM gathers the factor model specifications, namely $F M_{I C_{3}}$ and $F M_{S C}$; and BVAR aggregates the three Bayesian VAR models: BVAR $\mathrm{BC}_{\text {, }}, \mathrm{BVAR}_{0.2}$, and BVAR , $_{0 .}$. Percentages are also computed across the four datasets discussed in Section 5.1. Values greater or equal than $50 \%$ are highlighted in bold. 


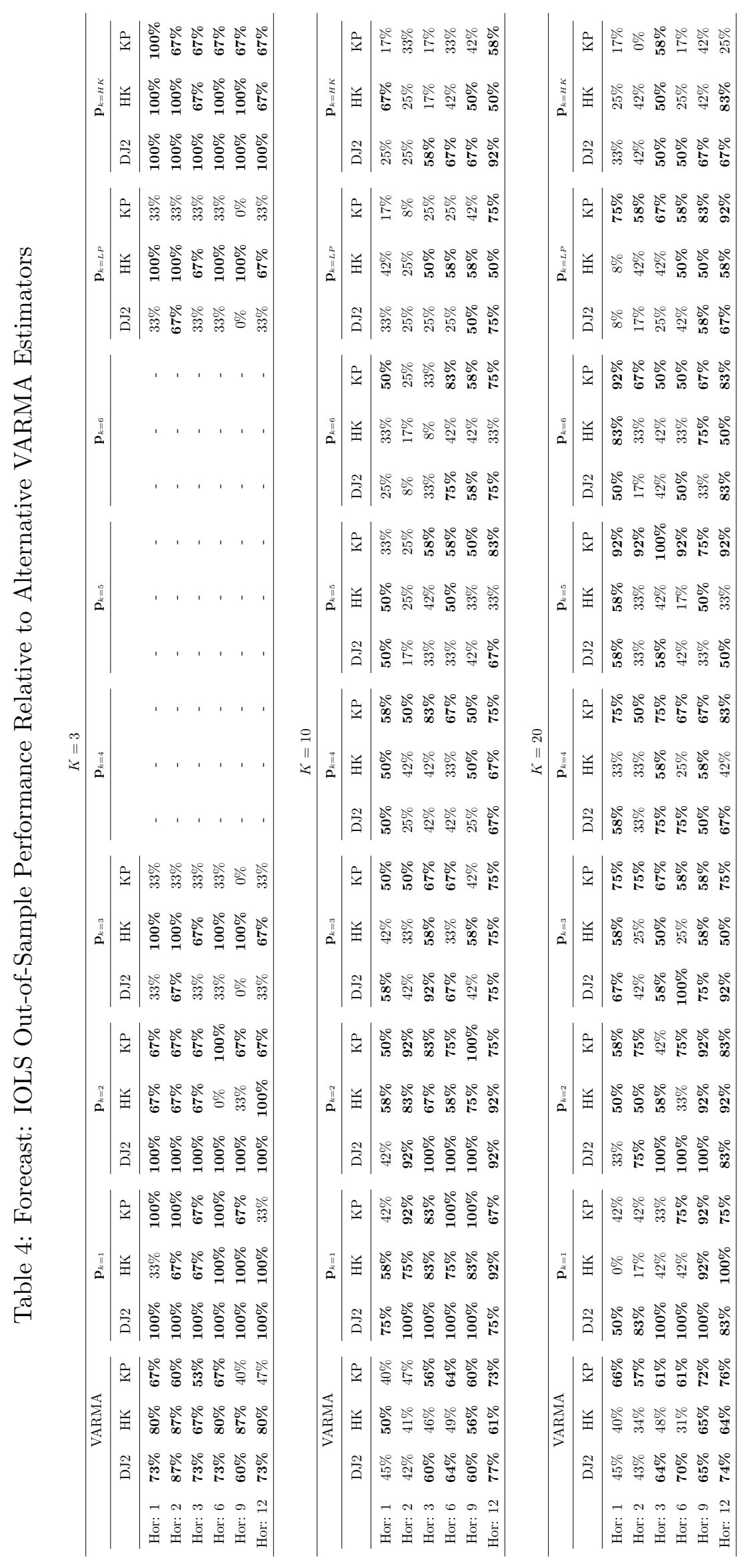

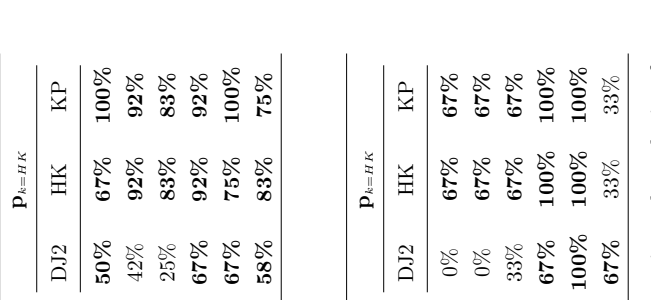

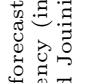

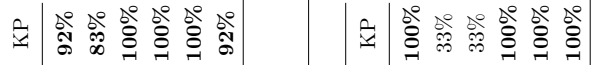

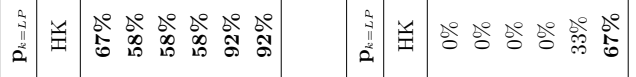

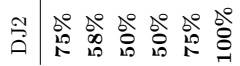

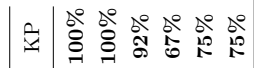

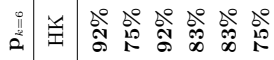

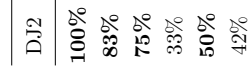

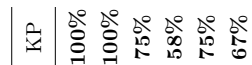

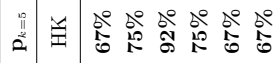

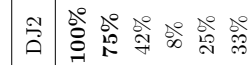

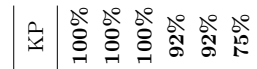

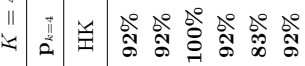

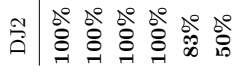

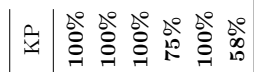

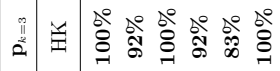

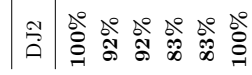

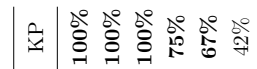

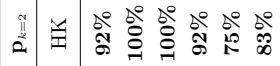

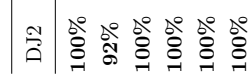

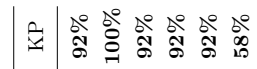

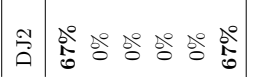

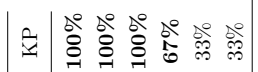

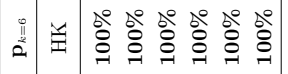

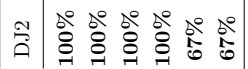

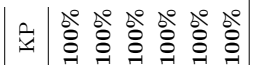

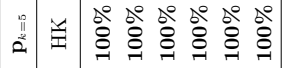

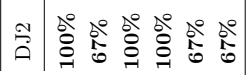

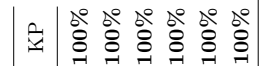

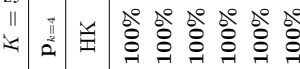

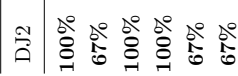

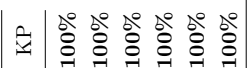

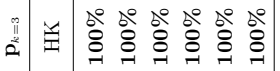

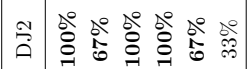

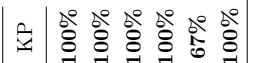

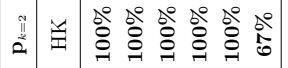

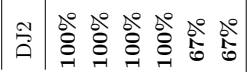

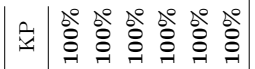

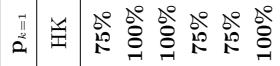

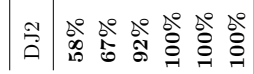

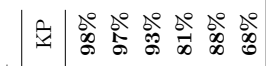

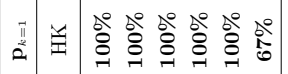

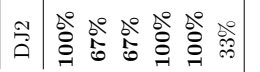

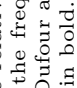

政

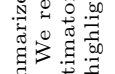

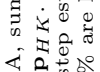

존

年

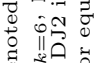

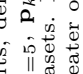

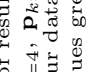

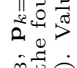

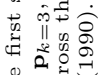

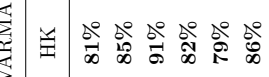

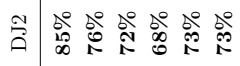

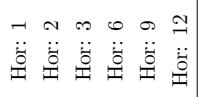

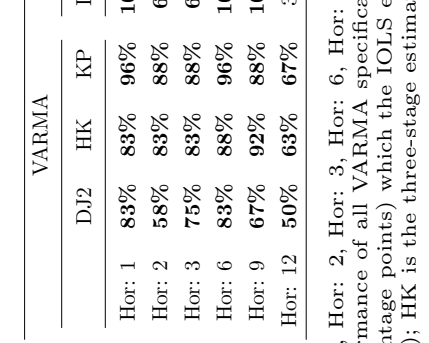

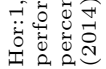

